OPEN ACCESS

Edited by:

Olli Tenovuo,

University of Turku, Finland

Reviewed by:

Istvan Jozsef Merchenthaler,

University of Maryland, Baltimore,

United States

Francisco Capani,

University of Buenos Aires, Argentina

${ }^{*}$ Correspondence:

Eduardo Martínez-Martínez emartinez@inmegen.gob.mx

Specialty section

This article was submitted to Neurotrauma

a section of the journa

Frontiers in Neurology

Received: 04 July 2020 Accepted: 11 November 2020 Published: 11 December 2020

Citation:

Quiroz-Baez R, Hernández-Ortega K and Martínez-Martínez E (2020) Insights Into the Proteomic Profiling of

Extracellular Vesicles for the Identification of Early Biomarkers of

Neurodegeneration.

Front. Neurol. 11:580030.

doi: 10.3389/fneur.2020.580030

\section{Insights Into the Proteomic Profiling of Extracellular Vesicles for the Identification of Early Biomarkers of Neurodegeneration}

\author{
Ricardo Quiroz-Baez ${ }^{1}$, Karina Hernández-Ortega ${ }^{2}$ and Eduardo Martínez-Martínez ${ }^{3 *}$ \\ 'Departamento de Investigación Básica, Dirección de Investigación, Instituto Nacional de Geriatría, Ciudad de México, \\ Mexico, ${ }^{2}$ Departamento de Genética del Desarrollo y Fisiología Molecular, Instituto de Biotecnología, Universidad Nacional \\ Autónoma de México, Cuernavaca, Mexico, ${ }^{3}$ Laboratory of Cell Communication \& Extracellular Vesicles, Division of Basic \\ Science, Instituto Nacional de Medicina Genómica, Ciudad de México, Mexico
}

Extracellular vesicles (EVs) are involved in the development and progression of neurodegenerative diseases, including Alzheimer's and Parkinson's disease. Moreover, EVs have the capacity to modify the physiology of neuronal circuits by transferring proteins, RNA, lipids, and metabolites. The proteomic characterization of EVs (exosomes and microvesicles) from preclinical models and patient samples has the potential to reveal new proteins and molecular networks that affect the normal physiology prior to the appearance of traditional biomarkers of neurodegeneration. Noteworthy, many of the genetic risks associated to the development of Alzheimer's and Parkinson's disease affect the crosstalk between mitochondria, endosomes, and lysosomes. Recent research has focused on determining the role of endolysosomal trafficking in the onset of neurodegenerative diseases. Proteomic studies indicate an alteration of biogenesis and molecular content of EVs as a result of endolysosomal and autophagic dysfunction. In this review, we discuss the status of EV proteomic characterization and their usefulness in discovering new biomarkers for the differential diagnosis of neurodegenerative diseases. Despite the challenges related to the failure to follow a standard isolation protocol and their implementation for a clinical setting, the analysis of EV proteomes has revealed the presence of key proteins with post-translational modifications that can be measured in peripheral fluids.

\footnotetext{
Keywords: extracellular vesciles, central nervous system, neurodegenenerative diseases, biomarkers, proteomic analyses, Parkinson' s disease, Alzheimer's disease, endolysosomal dysfunction
}

\section{INTRODUCTION}

Neurodegenerative diseases (NDs) are complex disorders with devastating consequences for the patient and their immediate social environment. In Alzheimer's disease (AD) and Parkinson's disease (PD), the neuronal dysfunction and secondary effects progress gradually with heterogeneous clinical outcomes. Despite the advances in management of $\mathrm{AD}$ and $\mathrm{PD}$, these diseases are among the leading causes of disabilities and showed an increased burden especially in low- and middle-income countries (1). One of the major challenges in the field of neuroscience is to achieve an early and precise detection of NDs to procure an adequate treatment and delay their 
progression. Most of the patients are diagnosed when overt symptoms of neurodegeneration are displayed. Furthermore, it has been documented that the misdiagnosis rate of $\mathrm{AD}$ and PD could be as high as 17 and $25 \%$, respectively (2-5). Thus, there is a need to develop new diagnostic strategies to aid in the identification of people developing $\mathrm{AD}$ and $\mathrm{PD}$.

The specific cause of neural cell death has not been resolved, but it has become clearer that in $\mathrm{AD}$ and $\mathrm{PD}$, both genetic and environmental factors operate. In the last two decades, several chromosomal regions have been identified containing genes that are associated with the risk of developing these NDs. In the early-onset variety of $\mathrm{AD}$ and $\mathrm{PD}$, a point mutation could be identified as a causal agent due to the impairment of the function of a specific protein. Among the most relevant genes that contain genetic variants for $\mathrm{AD}$ development are the APP, PSEN1, PSEN2, APOE, ADAM10, and ACE genes (6). In the case of $P D$, the development of the disease has been linked to the following genes: SNCA, PRKN, PINK1, DJ-1, LRRK2, ATP13A2, PLA2G6, FBX07, and VPS35 (7, 8). Despite early-onset cases representing $<10 \%$ of the total $\mathrm{AD}$ and $\mathrm{PD}$ cases, these studies have helped to determine the main processes that underlie neurodegeneration processes. In general, these two diseases are characterized by alterations in mitochondrial function, in management of oxidative stress, in protein folding and aggregation, and in immune function (7-9).

The diversity and severity of symptoms and the differences in the timing of neurodegenerative progression have made it difficult to create clinically applicable tests for the diagnosis of $\mathrm{AD}$ and $\mathrm{PD}$. In many cases, the confirmation of a ND is performed post-mortem. For this reason, the definition of a set of biomarkers is essential for diagnosis, stratification of patients and therapeutic monitoring. In recent years, the fact that altered protein homeostasis is a common event in the development of NDs has guided the development of diagnostic methods. For instance, several studies have found that decreased levels of amyloid $\beta$ peptide of 42 amino acids length $(\mathrm{A} \beta 42)$ in $\mathrm{AD}$ and of $\alpha$-synuclein ( $\alpha$-syn) in PD in cerebrospinal fluid (CSF) indicate an overt development of the disease, but low specificity and contradictory results have been reported $(10,11)$. Nevertheless, these findings set the precedent for the identification of circulant biomarkers indicating the progress of NDs. A relevant aspect in the development of $\mathrm{AD}$ and $\mathrm{PD}$ is the dysfunction of the interaction between endosomes, lysosomes, and mitochondria at various levels. These alterations modify the secretory capacities of the cells in the brain, which is reflected in the molecular composition of organelles released into the extracellular space, which are known as extracellular vesicles (EVs).

The generic term of EVs refers to the lipidic vesicles that are released from the endosomal system (exosomes) and from the plasma membrane (microvesicles or ectosomes) (Figure 1A). EVs are considered a novel system of intercellular communication that involves the transfer of proteins, RNA, lipids, and metabolites. An alteration of the molecular cargo of EVs has been associated with the development of several diseases. In the case of NDs, it has been proposed that these organelles are involved in the propagation of mis-folded proteins in the brain (12). Moreover, it has also been documented that part of the communication between glia and neurons is mediated by EVs and their alteration has implications for the development of NDs. The advantage of studying EVs is that exosomes and microvesicles can be isolated from fluids such as CSF, blood, saliva, or urine. Thus, the molecular characterization
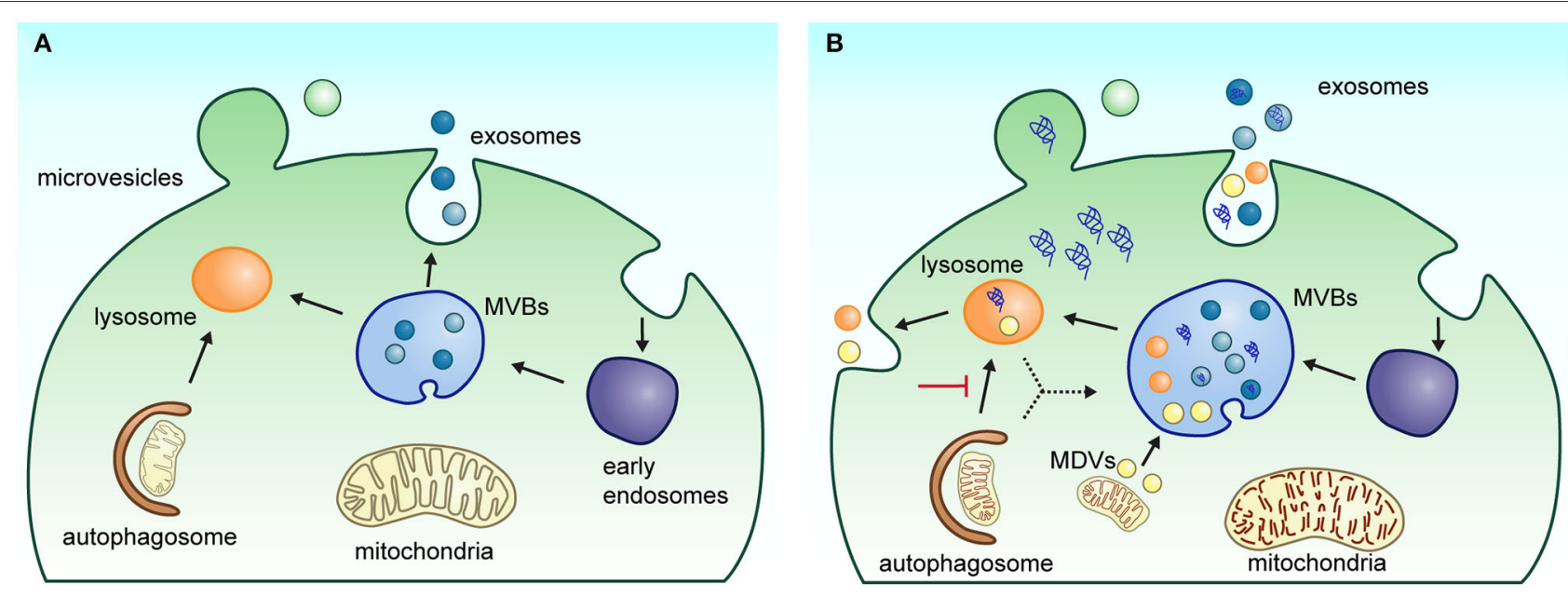

FIGURE 1 | Biogenesis and secretion pathway of EVs. (A) Under physiological conditions, extracellular vesicle biogenesis and secretion process occur in two main ways: (1) exosomes are generated in multivesicular bodies (MVBs) following the endolysosomal pathway; when MVBs fuse with the plasma membrane, exosomes are released into the extracellular space; or (2) microvesicles are released directly by budding from the plasma membrane. (B) Under neuropathological conditions such as Parkinson's and Alzheimer's disease, alterations in the endolysosomal system can affect biogenesis and release of EVs in the brain cells. The impairment of the autophagic-lysosomal pathway and alteration in protein sorting contribute to the increased accumulation of pathological markers in EVs. Several neurological diseases show an enlargement of MVBs with an increased number of intraluminal vesicles. The deficient mitophagy process also induces dysfunctional mitochondria accumulation, favoring the generation and release of mitochondrial-derived vesicles (MDVs) by cells. 
of EVs is promising because the analysis in both patient samples and disease models may reflect the type of molecules that are expressed at different stages of the development of NDs. Interestingly, it has been described that brain-derived exosomes can be found in peripheral circulation. In this review, we will focus on discussing the scope and limitations of the proteomic characterization of EVs and their potential to identify molecules that guide the development of non-invasive tests and to help unravel neurodegenerative pathophysiology.

\section{ROLE OF EVS IN THE CENTRAL NERVOUS SYSTEM}

The intercellular communication mediated by EVs has been linked to various processes of normal brain function both in development and in adulthood. During the synaptogenesis process and proliferation of neural progenitors, the gradients of hydrophobic molecules such as Wnt and Sonic the Hedgehog are generated by their release and transport in EVs (1315). For neural circuit formation, neuronal exosomes promote neuronal precursor proliferation and cell differentiation both in vivo and in vitro. The EVs derived from human induced pluripotent stem cells (iPSC) are enriched in proteins related to neurodevelopmental functions including neuritogenesis and morphology of the nervous system. These functions are impaired due to the loss of MECP2, which also alters synaptic density and neuronal firing capabilities (16). In the subventricular zone, the neural stem cells release vesicles that function as morphogens by decreasing the number of microglial processes to induce its traditional stellate morphology. In response, microglia can reduce the proliferation of neural precursors through cytokine release (17). It appears that EV-mediated communication is an active system that continues into adulthood. In ultrastructural studies, the presence of vesicles in the intercellular space in various brain structures is notorious, which suggests the existence of communication axes between the different cell types in the brain: neurons, astrocytes, microglia, and endothelial and epithelial cells $(18,19)$.

At a neuronal level, synaptic maturation and remodeling are influenced by the molecular cargo of EVs. For example, the degradation of the post-synaptic density protein-95 is promoted by the delivery of Proline-Rich 7, which results in the elimination of excitatory synapses in rat hippocampal neurons (15). The release of EVs from the presynaptic terminal is activity dependent and promotes the delivery of molecules to the post-synapsis such as Synaptotagmin-4, which, by retrograde signaling, promotes the maturation of the presynaptic terminal at the neuromuscular junction of Drosophila $(20,21)$. Likewise, synaptic plasticity is influenced by EV-mediated transfer of Arc1 mRNA to the post-synaptic region $(22,23)$. While epileptic status selectively modifies the miRNA content of hippocampus-derived EVs, it does not modify the process of miRNA editing that occurs inside the vesicle, which modulates target recognition (24). These findings suggest that the information contained in the EVs add an extra layer of modulation for normal synaptic functioning, which can be bidirectional among neurons.
Regarding the communication between different brain cell types, there is evidence that EVs are targeted to specific cell types. Selective expression of CD63 coupled to GFP in neurons has been achieved in rodent models. Neuron-derived EVs containing GFP are incorporated preferentially by astrocytes and also in a minor proportion by certain neurons, both in vivo and in vitro experiments $(25,26)$. These vesicles are mainly secreted at the neuronal soma and dendrites, and they transfer the miR-124$3 p$, which in turn increases protein levels of the glutamate transporter GLT1 in astrocytes (26). Astrocyte-derived EVs seem to be directed mainly toward neurons to modulate a variety of functions (27). The effect of astrocyte-derived EVs depends on the brain microenvironment, being beneficial when there are trophic stimuli or neurotoxic when there are proinflammatory conditions. After incubating astrocytes with ATP, the EVs are enriched with proteins related to synaptogenesis (NETO1) and neurite growth (RPL10). In contrast, astrocyte stimulation with IL-1 $\beta$ or TNF- $\alpha$ enriches proteins and miRNAs associated with reduced growth and network neuronal activity $(28,29)$. In addition, the activation of astrocytes by IL- $1 \beta$ is associated with the production of vesicles that are released into peripheral circulation and that promote migration of leukocytes into the brain (30). In the case of microglia, an interaction with neurons through EVs has also been documented. The incubation of the MG6 microglial cell line with EVs from depolarized neurons promotes the degeneration of neurites from PC12 cells (31). A deleterious effect is also observed with the EVs derived from microglia BV2 cells after the activation of the metabotropic glutamate receptor 5, which is involved in neuroinflammation (32). LPS increases the levels of TNF- $\alpha$ and IL-6 in microglia-derived EVs and promotes the loading of proteins related to transcription and protein translation (33). Further research is needed to determine the role of microgliaderived EVs in normal conditions and after traumatic brain injury, which induces changes in miRNA cargo that have been related to anti-inflammatory effect on neurons (34).

Endothelial cells constitute another important participant of the intercellular communication in the brain. The survival and proliferation of oligodendrocyte precursor cells are promoted by EVs derived from microvascular endothelial cells (35). Likewise, endothelial cell-derived EVs inhibit apoptosis of neurons deprived of oxygen and glucose through the transfer of miR-1290 (36). Endothelial cells can also be influenced by astrocyte-derived vesicles that regulate the expression of tightjunction proteins (claudin-5, occluding, ZO-1) to maintain the integrity of the blood-brain barrier (37). After TNF- $\alpha$ exposure, the protein related to TNF signaling, immune response, and mitochondrial proteins are enriched in the EVs derived from endothelial cells (38). These findings indicate that EV secretion by endothelial cells has a role in the activation of an inflammatory systemic response. Indeed, a focal brain lesion induced by a microinjection of IL-1 $\beta$ in the brain parenchyma increases the circulating EVs from endothelial cells and induces the release of acute-phase response proteins by the liver (39). Conversely, the brain can be exposed to signals from the periphery. Breast cancer-derived EVs can cross the blood-brain barrier through a transcellular mechanism that involves the downregulation of 
Rab7 to switch the endocytic pathway to a recycling mode (40). The choroid plexus epithelium is another sensor of peripheral conditions that can modify brain cell physiology. After LPS injection in mice, choroid plexus epithelial cells release EVs into the CSF containing inflammatory miRNAs (miR-1a, miR-9, miR-146a, and miR155) that eventually activate an inflammatory response in astrocytes and microglia (19).

Because CSF contains a mixture of molecules originating from different cells in the brain, it has been used to monitor the physiological status of the CNS. In relation to EVs, there is evidence showing that the isolation of vesicles from CSF could be informative about the progression of neurological diseases. Recently, the analysis of patient-derived glioblastoma cells revealed that a subpopulation of EVs is mainly enriched in mRNA and non-vesicular ribonucleoprotein complexes are enriched in tRNAs and Y RNA fragments $(41,42)$. These changes can be detected by the analysis of EVs in CSF samples and help to provide a molecular diagnosis (43). The principle of monitoring pathological events in the brain is reinforced by the fact that EVs derived from peripheral blood reflect the presence of biomarkers of the developing tumor in the brain and its levels correlate with cell invasiveness $(41,42,44)$. Similarly, the protein cargo of EVs from blood and CSF samples reflects early events of neurodegeneration related to mild traumatic brain injury and cognitive impairment (45-47). These proteomic profiling studies indicate alterations in the endosomal and lysosomal functions which are key processes underlying NDs. A possible role of EVs in the brain is to discard an overload of mis-folded proteins to maintain synaptic homeostasis $(48,49)$.

\section{OVERVIEW OF THE ROLE OF EVs IN DEVELOPMENT AND PROPAGATION OF NDS}

Recently, it has been suggested that the classification of EVs in exosomes and microvesicles is an oversimplification of the heterogenous mixture of EVs that originate from different intracellular pathways $(50,51)$. While the exact composition and function of each EV subtype is still not well-defined, the current available methods for its molecular characterization indicate that a set of molecules associated with the development of NDs can be enriched or depleted under certain conditions. These changes may result from an alteration in the biogenesis of EVs or from a neuronal response to eliminate protein aggregates to counteract impairments in lysosomal and autophagy functions. In agreement with this notion, it has been observed that in several models of neurodegeneration, there are morphological alterations in the endolysosomal system, including larger multivesicular bodies (MVBs) with more intraluminal vesicles (52-54). In addition, neurodegeneration is related to changes in the expression of endolysosomal markers (EEA1, Rab5B, Rab7, CD63, TSG101, Rab35, LAMP1, and CTSD) and lipid metabolism (sphingolipids and lysobisphosphatidic acid) $(49,54$, 55). Neurons with a reduced capacity of protein and organelle degradation are prone to accumulate protein aggregates, which apparently are propagated through EV release (Figure 1B).
Histological, biochemical, and proteomic analysis of EVs in different research models and patient samples have shown that $\mathrm{A} \beta$ and Tau in $\mathrm{AD}$, and $\alpha$-syn in PD are associated with EVs (56). In human brain sections from $\mathrm{AD}$ patients, the exosome marker flotillin-1 colocalizes with oligomers of $A \beta$ (57). The injection of EVs isolated from familial AD patient-derived iPSCs in mouse hippocampus was sufficient to induce Tau phosphorylation in different regions of the hippocampus after 5 weeks (58). The Swedish mutation in the amyloid precursor protein (APP), which increases abnormal cleavage of cellular APP, favors the loading of $\mathrm{A} \beta 40, \mathrm{~A} \beta 42$, and APP C-terminal fragments (CTFs) into EVs that are incorporated mainly by neurons (59). In an elegant study, it was shown that the disruption of the PI3K/Vps34 signaling pathway is involved in the secretion of APP-CTFs by causing a dysregulation of lipid metabolism that induces damage to endolysosomal membranes (49). A reduced EV release has been observed in a humanized APOE4 mouse model. The presence of the risk allele of apolipoprotein $\mathrm{E}$ is associated with decreased levels of Tsg101 and Rab35 (55). These findings are of great relevance considering that genetic risk factors of NDs usually involve proteins of endosomal and lysosomal trafficking. A compromised ability to handle cellular waste by the disturbance of endolysosomal trafficking can be reflected in the molecular content of EVs.

Similar evidence has been described for PD in which the dysfunction of key elements of lysosomal and endosomal pathways has been associated with the development of the disease (7). An analysis of PD human brain extracts showed that $\alpha$-syn accumulation is associated with decreased levels of the lysosomal protein LAMP2 (60). For instance, the loss of the mitochondrial protein Parkin (PARK2) decreased the endosomal tubulation and increased the expression of late endosome markers such as CD63 and the number of intraluminal vesicles in MVBs (61). The overexpression of the lysosomal protein ATP13A2 (PARK9) increases its presence in MVBs and promotes the secretion of $\alpha$-syn in EVs (62). Interestingly, the inhibition of the enzyme neutral sphingomyelinase (nSMase2), involved in the biogenesis of intraluminal vesicles, decreased the transfer of oligomeric aggregates of $\alpha$-syn in co-culture experiments (63). The leucinerich repeat kinase 2 (LRRK2) is naturally found in EVs from kidney, immune, and brain cells, and it is proposed to have a key role in autophagocytic pathway $(64,65)$. Levels of phosphorylated LRRK2 at residue S1292 in CSF and urine correlate with severity of the disease and help to predict mutation carriers (66). Overall, an early event in protein aggregation seems to involve components of the endosome and lysosome compartments.

\section{EV PROTEINS AS BIOMARKERS IN PD}

PD is characterized by non-motor and motor alterations that are associated with the progressive loss of dopaminergic neurons in the substantia nigra pars compacta. A well-established histological feature is the presence of cytoplasmic inclusions known as Lewy bodies (LBs) that are composed of $\alpha$-syn aggregates and sequestered organelles including mitochondria, endosomes, and lysosomes (67). Growing evidence indicates that 
$\alpha$-syn pathology initiates in the periphery even earlier than in the central nervous system $(68,69)$. The mechanisms considered for $\alpha$-syn pathology spreading include axonal transport, fluid phase, prion-like process, and EV-mediated transmission (70). Considering that EVs reflect cellular changes that occur in response to pathological conditions, it is conceivable that they are an effective source of biomarkers for both peripheral and central events of neurodegeneration.

Among the intracellular mechanisms proposed to explain PD pathogenesis, included is the disturbance of the interactions between mitochondria, lysosomes, and endoplasmic reticulum, which results in the accumulation of protein aggregates and cellular toxicity. The deletion of mitochondrial proteins (AIF, OPA, and PINK1) or inhibition of mitochondrial complex I with rotenone cause the appearance of lysosomal vacuoles, increase lysosomal permeability, and alter protein degradation $(71,72)$. The absence of PINK1 and Parkin induces the formation of mitochondrial-derived vesicles that can be shuttled to late endosomes and secreted to extracellular space (73). Similarly, the inhibition of the maturation of autophagosomes with bafilomycin promotes their fusion with multivesicular bodies and favors the release of $\alpha$-syn through EVs (74). Altogether, these data indicate that dysfunction of organelle crosstalk not only creates a microenvironment for protein aggregation but also alters the dynamics of protein cargo that is packaged into EVs.

The protein $\alpha$-syn is expressed in the brain and is present in an oligomeric or aggregated state in body fluids, CSF, and plasma (75). The levels of $\alpha$-syn in EVs from CSF allow the discrimination of patients with PD and dementia with LBs from other neurological conditions and healthy controls (76). To achieve a better resolution, EVs from the central nervous system have been isolated by immunocapture using an anti-L1CAM antibody, which recognizes a cell adhesion marker enriched in neurons (77-79). The amount of neuron-derived EVs and $\alpha$ syn levels in these EVs is higher in plasma of PD patients than in plasma of healthy controls $(77,79)$. A relevant point to mention is that measurement of $\alpha$-syn in EVs from a specific cell population could provide a more consistent assessment of the quantity of a biomarker at different stages and types of NDs than measurements directly from total CSF or plasma. A crosssectional study showed that $\alpha$-syn in neuronal EVs is increased by two-fold in prodromal and clinical PD in comparison to multiple system atrophy, controls, or other NDs (80). Combined evaluation of $\alpha$-syn and clusterin in neuron-derived EVs improves the differential diagnosis to predict PD from non- $\alpha$ syn proteinopathies (80). Furthermore, an increased proportion of oligodendrocyte-derived EVs and astrocyte-derived EVs in relation to neuron-derived EVs in plasma shows a significant correlation with the Unified Parkinson's Disease Rating (UPDRS) part III scores in the patients with PD (79). Taken together, these data suggest that EVs derived from the central nervous system would be a helpful strategy with elevated specificity and sensitivity to differentiate and monitor the progression of PD.

The presence of relevant biomarkers in EVs has also been studied in other biofluids. In a small sample of Korean PD patients, $\alpha$-syn was not detected in urine EVs (81). In contrast, the levels of $\alpha$-syn oligomers and the ratio of $\alpha$-syn oligomers to total $\alpha$-syn is increased in salivary EVs isolated from PD patients (82). Additionally, the concentration of salivary EVs of neuronal origin is higher in PD patients than in control individuals, as well as the levels of phosphorylated $\alpha$-syn (83). Tear fluid is also accessible and mirrors the pathophysiological changes in systemic and ocular diseases. A mass spectrometry analysis of tear samples in a small cohort of PD patients revealed that almost half of the deregulated proteins in PD are related to neuronal functions. Remarkably, DJ-1 levels are increased in PD tear samples vs. control tears (84). Dysregulation of core networks of proteins involved in lipid metabolism, oxidative stress, vesicle secretion, and immune response supports the participation of these mechanisms in PD pathogenesis (84). Further studies, including proteomic studies of tear fluid EVs in PD, are needed to deeper evaluate them as a feasible biomarker source for PD.

Regarding the observation of an altered EV content and secretion in PD patients, it can be correlated to impaired lysosomal function. Decreased glucocerebrosidase 1 (GBA-1) enzymatic activity in the brain is a common trait in PD. Dysfunction of the endocytic pathway through the inhibition of GBA-1 results in increased release of brain EVs containing $\alpha$-syn oligomers $(85,86)$. The increased production of $\mathrm{EV}$ associated with $\alpha$-syn contributes to accelerate protein aggregation in receptor cells in vivo (85). GBA1-associated neurodegeneration in PD is associated with impairment of the autophagiclysosomal pathway and mitochondrial dysfunction, possibly attributable to the accumulation of dysfunctional mitochondria as a consequence of defective mitophagy (87). Moreover, $\alpha$-syn aggregation can be involved in the dysregulation of autophagic and endolysosomal pathways and is also associated with mitochondrial dysfunction (88).

Mutations in the genes encoding for LRRK2 (PARK8) and DJ1 have been associated with PD. The mutations in the kinase LRRK2 account for $40 \%$ of the familial cases and its loss of function alters the endolysosomal pathway. The most prevalent mutation LRRK2-G2019S interferes with the mitochondrial fission through increasing the expression of the factor DLP1 (89). Regarding the possible role of these proteins as biomarkers, it is known that the levels of DJ-1 and LRRK2 in urine-derived EVs differ between sexes (81). Notably, DJ-1 levels increase with age in PD male patients (81). A high ratio of Ser(P)-1292 LRRK2 to total LRRK2 in urine EVs seems to be useful in discriminating between LRRK2 mutation carriers and non-carriers with or without PD. Moreover, LRRK2 mutation carriers with PD have a higher ratio than control individuals (66). Also, Ser(P)-1292 LRRK2 levels correlate with the severity of cognitive impairment and difficulty in accomplishing activities of daily living (90). While its exact function in PD pathogenesis is not fully understood, DJ1 may act as an antioxidant and chaperone and play a role in mitochondrial homeostasis, possibly involving the PINK1/Parkin pathway. The levels of DJ-1 in EVs derived from CNS and the ratio of EV DJ-1 to total DJ-1 are substantially higher in the plasma of PD patients compared to healthy controls (91). These data show promising results regarding the quantification of LRRK2 and DJ-1 in EVs and considering post-translational modifications could increase the accuracy for PD diagnosis and prognosis. 
Biomarker discovery can be accelerated by the characterization of EVs using mass spectrometric analysis for the high-throughput identification of dysregulated proteins in NDs. Despite the challenges of EV isolation from a reduced sample volume and the purity of EV preparations, shotgun proteomic profiling experiments have started to reveal the families of molecules that are associated with EVs. A total of 1,033 proteins were identified in a proteomic analysis of EVs isolated from sera of healthy controls and PD patients in early stages of the disease. Of these, 21 proteins were upregulated and 2 were downregulated in PD samples, while the quantity of vesicles and the amount of classical exosome markers such as flotillin were similar (92). Among the most abundant proteins are the vacuolar protein sorting-associated protein 13D, peroxiredoxin-2, S100A8, cytochrome b-245 heavy chain, and syntenin 1 (92). A more refined analysis using neural immunocaptured EVs from serum identified 429 proteins samples from controls and patients with mild (Hoehn and Yahr score < 3) and severe PD (Hoehn and Yahr score > 3) (93). The upregulated proteins in mild and severe cases include the clusterin, complement C1r subcomponent, afamin, apolipoprotein D, gelsolin, and pigmented epitheliumderived factor (PEDF). The down-regulated proteins in serum-derived EVs comprise human neuroblastoma cDNA clone CS0DD006YL02, complement C1q subcomponent, myosin-reactive immunoglobulin, Ig kappa chain, and Ig mu chain (93). In contrast, the proteomic characterization of plasma-derived EVs isolated by size exclusion chromatography showed that the levels of clusterin and C1r subcomponent were decreased in PD patients compared to control individuals (94). These disparate results highlight the importance of the isolation method and the need to determine whether differentially abundant proteins correspond to vesicular or extravesicular material.

Recently, a strategy to define the origin of EV subpopulations in blood was generated through the phenotyping of EVs by flow cytometry. The mean number of erythrocyte-derived EVs $\left(\mathrm{CD} 235 \mathrm{a}^{+}\right)$correlated with the UPDRS scores for different disease stages. The proteomic analysis of EVs from erythrocytes revealed that 8 proteins of a total of 818 identified proteins showed significantly different levels as a function of $\mathrm{PD}$ stage (95). The protein markers that were highly expressed in controls include axin interactor dorsalization-associated protein (AIDA), alpha/beta hydrolase domain-containing protein $14 \mathrm{Bv}(\mathrm{ABHD} 14 \mathrm{~B})$, and glutamine-dependent $\mathrm{NAD}^{+}$ synthetase (NADSYN1), while proteins highly expressed in mild PD were quinoid dihydropteridine reductase (QDPR), alcohol dehydrogenase $\mathrm{NADP}^{+}$(AKR1A1), and cannabinoid receptor-interacting protein 1 (CNRIP1). In contrast, the ubiquitin carboxyl-terminal hydrolase 24 (USP24) and ATP synthase subunit alpha mitochondrial (ATP5A1) were increased in moderate PD cases (Hoehn and Yahr score 2-2.5) (95). Future studies should explore differential protein signatures in other blood cell types such as immune cells, considering the relevance of persistent inflammation as a risk factor for developing PD.

The isolation of EVs from plasma, serum, and CSF samples represents several limitations including the limited volume obtained per patient and the technical procedures for its obtention. Conversely, urine samples can be used to maximize the yield of EVs and still capture the pathological processes occurring in different parts of the body. Consistent with this notion, a quantitative analysis of urinary EVs from a cohort of $\mathrm{PD}$ patients revealed that enriched proteins are related to neurological disorders including Parkinson's, Huntington's, and Alzheimer's diseases (96). Among the enriched endolysosomal proteins related to NDs, synaptosomalassociated protein 23 (SNAP23) and calbindin were particularly prominent in $\mathrm{PD}$ cases. The measurement of these proteins have a prediction success in a range of $76-86 \%$ for disease diagnosis in two independent cohorts (96). Moreover, this study demonstrated that EV-related proteins show low interindividual variability and can be tracked over time. Overall, these findings open the possibility of monitoring long term the abundance of proteins associated with neurological disorders and how their levels are modified in response to treatments.

Mitochondrial dysfunction causes oxidative stress favoring aberrant protein folding and protein accumulation (i.e., $A \beta$, huntingtin, Tau, and $\alpha$-syn). Furthermore, mitochondrial damage and impairment of the removal of damaged mitochondria (mitophagy) have been proposed as a central event in the pathogenesis of PD. The analysis of circulant mitochondrial-derived vesicles can be promising for the identification of early biomarkers of PD, considering that it is a cellular mechanism of antigen presentation in inflammatory conditions (73). A recent characterization of small EVs from the serum of PD patients identified a reduction in the levels of mitochondrial markers such as adenosine triphosphate 5A (ATP5A), NADH: ubiquinone oxidoreductase subunit S3 (NDUFS3), and succinate dehydrogenase complex iron sulfur subunit $\mathrm{B}$ (SDHB), and of the levels of the tetraspanins CD9 and CD63 (97). A more precise protein signature of mitochondrial-derived vesicles should be addressed in future studies including genetic models of PD and patient samples. A better characterization of the temporal dynamics of mitochondrial markers in circulation would help to understand the interconnection with the endolysosomal system.

The studies described above account for the most recent advances in the identification of prospective protein biomarkers contained in EVs. However, some points need to be resolved before a valid biomarker profile based on EV characterization can be efficiently used for the diagnosis and prognosis of PD. Ideally, an exhaustive phenotyping of EV subtypes could improve the detection of altered proteins related to neurodegeneration. Moreover, a multicenter study that guarantees the standardization of the protocols used for $\mathrm{EV}$ isolation and proteomic analysis would benefit the phase of validation of biomarkers in large size cohorts. Finally, as the familial forms of PD are associated with endolysosomal or mitochondrial defects, a targeted analysis of proteins associated with these cellular pathways in the EVs derived from body fluids of PD patients would be of great value in understanding the 
evolution of the disease and determining its relation to pharmacological treatments.

\section{EVs PROTEINS AS BIOMARKERS IN AD}

The histopathological hallmarks of $\mathrm{AD}$ are the presence of extracellular amyloid- $\beta$ (A $\beta$ ) plaques and intracellular neurofibrillary tangles composed of hyperphosphorylated Tau protein. The exact mechanism that provokes this malignant protein aggregation is not fully understood. In the development of AD, EVs show a multifaceted role. Several studies have demonstrated a deleterious effect of EVs as carriers of pathogenic proteins that contribute to their spreading across the brain. However, others state that it is possible that EVs have a neuroprotective role by buffering the formation of toxic protein aggregates. The specific function of brain EVs seems to depend on their cellular origin and on the inflammatory status of the brain.

Neuroblastoma N2a-derived EVs act as scavengers of neurotoxic forms of $\mathrm{A} \beta$ by trapping the peptides on its surface through glycosphingolipids. These $\mathrm{A} \beta$-EV complexes can be internalized by microglia for degradation (98). Moreover, the intracerebral infusion of EVs isolated from neuroblastoma cells diminishes $A \beta$ pathology and amyloid deposition in APP transgenic mice (99). These findings suggest the use of EVs as a novel therapeutic approach to preventing plaque deposition in $\mathrm{AD}$. In support of this notion, it has been proposed that the administration of EVs from hypoxic mesenchymal stem cells (MSCs) improves the learning and memory capabilities of the APP/PS1 mice by restoring the synaptic function. The EVs from hypoxic MSCs reduce the glial activation through the increase of anti-inflammatory cytokines (IL-10, IL-4, IL-6, and VEGF) and the decrease of proinflammatory cytokines (TNF- $\alpha$ and IL-1 $\beta$ ) concomitantly via a reduction in the activation of the STAT3 and NF-KB pathway $(100,101)$. The administration of MSC-EVs also influences presynaptic functions by the induction of longterm potentiation (cellular correlate of learning and memory) at Schaffer collateral to CA1 synapses, which improves cognitive behavior (102). MSC-EVs also protect neurons by reducing oxidative stress through the transfer of catalase and by promoting synapse integrity (101).

An additional beneficial effect of EVs has been postulated through the transport of the cellular prion protein (PrPC), which is a glycoprotein with high affinity for the oligomeric form of the A 342 . The EVs purified from N2a, SHSY-5Y, and cortical neurons, highly enriched in PrPC, show a higher binding affinity for dimeric, pentameric, and oligomeric $A \beta$ species $(103,104)$. The EV-PrPC complex plays a protective role in sequestering soluble oligomeric forms of $A \beta$, reducing its neurotoxic effects by accelerating its fibrillization (103). A similar mechanism could also occur in vivo as the intracerebroventricular injection of human neural EVs in the brain rat prevents the inhibition of long-term potentiation caused by $A \beta$ oligomers (105).

The notion of a neurotoxic role of EVs has been based on data showing the release of pathological forms of $A \beta$ in the extracellular space by microglial and neuronal cells. As suggested by studies in $\mathrm{AD}$ animal models, EVs can diffuse throughout the brain and play a role in the dynamics of amyloid deposition (106). After $A \beta$ internalization into microglia, fibrils can be converted into an oligomeric toxic form and reintroduced into endosomal secretion pathway $(107,108)$. In neurons, the loss of endosomal sorting complexes required for transport (ESCRT) components or the ubiquitination factor E4B (UBE4B) increases the levels of $A \beta 42$ in late endosomes and its secretion through EVs (109). Both in vivo and cell culture assays have demonstrated that the dysfunction of the enzymes ECE-1 and-2, located in MVBs, could lead to the accumulation of intraneuronal $A \beta$ aggregates and their subsequent release through EVs (110). Thus, mutations or alterations in the molecular machinery involved in APP trafficking can favor the loading and secretion of EVs with toxic aggregates into the circulation. Interestingly, the measurement of $A \beta$ peptides and Tau levels in plasma-derived EVs has shown promising results in designing a score to follow $\mathrm{AD}$ progression and to discriminate from other types of dementias. With a sensitivity of 96\%, the levels of phospho-Tau (pTau)-T181, pTauS396, and $A \beta 42$ levels in neuronal EVs discriminate AD patients from match-case controls. Moreover, EV levels of $A \beta 42$ are an indicator of AD progression in preclinical cases (111).

The molecular characterization of EVs indicates that there is a basic set of proteins with a complex dynamic overtime, consisting of subtle changes in protein concentration and posttranslational modifications. Mild cognitive impairment has been associated with lower levels of total Tau and APP and a higher ratio of pTau-T181/total Tau in comparison to controls. AD can be discriminated from healthy individuals considering the ratio of pTau-T181/total Tau, and from mild cognitive impairment considering the pTau-T181/total Tau ratio and the APP levels in plasma-derived EVs. In comparison to controls, the APP levels are lower in mild cognitive impairment and in mild and moderate $\mathrm{AD}$, while high levels of APP characterize severe AD (112). These data indicate that the protein signature of EVs changes gradually during the development of AD. Other research groups have explored neuronal-derived EVs from plasma, the expression of transcriptional factors involved in neuronal defense against diverse stresses on $\mathrm{AD}$ patients. EVs of $\mathrm{AD}$ patients contain low levels of the low-density lipoprotein receptor-related protein 6 (LRP6), heat-shock factor-1 (HSF1), and repressor element 1silencing transcription factor (REST). These transcription factors are diminished 2-10 years before the clinical diagnosis of $\mathrm{AD}(113)$.

In $\mathrm{AD}$, the increased production of EVs is associated with the progression of Tau pathology $(111,114)$. The inhibition of exosome synthesis in microglia significantly reduces Tau propagation in vitro and in vivo (115). It is possible that EVs can contain different Tau species, including monomers, oligomers, and aggregates. However, only the aggregated forms can promote the aggregation of new Tau molecules. The release of EVs containing Tau is promoted by neuronal activity and thus may contribute to the spreading of Tau pathology through trans-synaptic transmission (116). EVs from the Tg4510 mouse (carrying the P301L Tau mutation) contain high levels of Tau with an altered pattern of Tau-phosphorylation (AT8, AT100, and AT180) that promote the formation of Tau inclusions. The P301L Tau-containing EVs transport Tau seeds, which are able 
to induce the aggregation of endogenous Tau in recipient cells, supporting the active role of exogenous seeds in nucleating nascent Tau inclusion through vesicle transport (117). Genomewide association studies suggest a strong connection of lateonset $\mathrm{AD}$ with Bridging INtegrator 1 (BIN1), which is involved in endosomal trafficking (118). In animal models and CSF of $\mathrm{AD}, \mathrm{BIN} 1$ is related to Tau secretion via EVs and could contribute to Tau pathology by altering Tau clearance and promoting the release of Tau enriched EVs by microglia (119). The characterization of EVs from human iPSCs, CSF, and plasma shows that the main type of Tau inside of EVs is the full length form, suggesting a dysfunction of autophagy (120). The presence of P301L and V337M Tau mutations in iPSC dysregulates the EV proteome. Some of the proteins exclusively present in mutant Tau-EVs have been reported to participate in cellular processes related to synaptic dysfunction, memory loss, and neuropathology. Remarkably, mutant Tau-EVs contain ANP32A, which is an endogenous inhibitor of protein phosphatase-2A (PP2A) that in turn regulates the dephosphorylation of Tau (121). Therefore, common mutations related to $\mathrm{AD}$ pathogenesis that alter endolysosomal trafficking may result in a noxious molecular cargo in EVs that contributes to the spread of toxic protein aggregates.

One of the major genetic risks associated with the development of late-onset $\mathrm{AD}$ is the allele $\varepsilon 4$ of the apolipoprotein E (APOE4). The expression of APOE4 is associated with decreased levels of EVs in the human brain and in APOE4 transgenic mice. The exosomal biogenesis pathway seems to be compromised by the presence of APOE4, as indicated by the downregulation of the transcription and translation of Tsg101 and Rab35 in the humanized APOE mice (55). Additional effects related to APOE4 include enlarged endosomes, cholesterol accumulation, and increased secretion of $\mathrm{A} \beta 42(55,122)$. The APOE4 genotype may contribute to the disruption of endosomal-lysosomal system function, probably due to disturbances in lipid metabolism that increase neuronal vulnerability over time. The exact mechanism has not been resolved, but it could involve a vicious cycle between astrocytes and neurons that ends in an inability of $A \beta 42$ clearance and altered EV molecular cargo. A mass spectrometry analysis of the EV content from a mixed co-culture of mouse primary astrocytes, neurons, and oligodendrocytes found that the exposure to $\mathrm{A} \beta 42$ protofibrils altered the levels of ApoE, $2^{\prime}, 3^{\prime}$ cyclic-nucleotide $3^{\prime}$-phosphodiesterase (CNPase), heavy chain 1 , $60 \mathrm{~S}$ ribosomal protein $\mathrm{L} 4$, and cytoplasmic dynein 1 heavy chain 1 proteins (123). Previously, the same group showed that the inability of astrocytes to clear $A \beta$ leads to the increased release of truncated forms of $\mathrm{A} \beta$ in EVs, thus inducing neural apoptosis (124). In line with these studies, an altered EV generation has been proposed from mass spectrometry analysis of neocortical brain tissue samples from $\mathrm{AD}$ patients. A pathway enrichment analysis revealed changes in proteins related to exocytic and endocytic pathways. Interestingly, among the proteins with higher levels in $\mathrm{AD}$ compared to control include EV-related markers such as CD9, HSP72, PI42A, TALDO, and VAMP2 (125). EVs from CSF and plasma of AD patients and AD mouse models expressing a presenilin-1 mutation show an increased
$\mathrm{A} \beta 42 / \mathrm{A} \beta 40$ ratio and impair $\mathrm{Ca} 2+$ handling and mitochondrial function (126). These findings suggest that distorted intercellular communication may contribute to neuronal dysfunction and highlights the role of glia in maintaining a healthy neuronal state.

Recent evidence also indicates the potential role of EVs in the transport of APP and its catabolites (CTFs). Mouse N2a cells expressing the Swedish mutation of human APP show a differential loading of CTFs into EVs. The Swedish mutation enriches CTF- $\alpha$ and CTF- $\beta$, but not CTF- $\eta$, into a subset of EVs that lack CD63. This subpopulation of EVs carrying CTFs only bind to dendrites of neurons, while $\mathrm{CD}^{+} 3^{+}$ EVs are targeted to both neurons and glial cells (59). These changes in composition and cellular selectivity may result from an impairment of the endolysosomal function that modifies lipid composition, membrane proteins type, and protein glycosylation. The chemical inhibition or genetic ablation of the kinase Vps34 results in endosomal abnormalities and autophagic blockage in primary cortical neurons and N2a cells (49). The EVs collected from these cultures are enriched in CTFs, in cholesterol and sphingolipid subclasses (dihydrosphingomyelin, monohexosylceramide, and lactosylceramide), and in the phospholipid bis(monoacylglycerol)phosphate. Furthermore, the global protein glycosylation is modified in specific brain regions of $\mathrm{AD}$ patients, and some of these changes are reflected in serum samples (127). An abnormal protein glycosylation pattern has been associated not only with $\mathrm{AD}$ development, but also with a differential cellular uptake of EVs (128-130). The fact that APP-CTFs and A $\beta$ are selectively sorted into a subpopulation of EVs and are specifically endocytosed by neurons suggests the mechanism in which EVs contribute to the spread of pathological fragments throughout the brain. In agreement with this notion, EVs isolated from human iPSCderived neuronal cultures harboring the A246E presenilin-1 mutation can induce Tau hyperphosphorylation. Although the mechanism remains unknown, the injection of these EVs into the CA1 region of the mouse hippocampus generates Tau aggregation in the hippocampus after 5 weeks $(58,131)$. Likewise, EVs from mice containing Tau with the familial dementia mutation P301L accelerate Tau phosphorylation and soluble aggregate formation in vivo (132). These findings support the role of EVs in the spreading of Tau pathology in AD, but long-term studies are required to determine the intracellular mechanism by which EVs induce Tau phosphorylation and whether there is a direct participation in neurofibrillary tangle formation.

A dysfunctional endosomal system is an early feature of neurons of individuals with Down syndrome (DS), which includes larger endosomes and an accumulation of APP metabolites in endosomal vesicles. EVs isolated from the brains of DS patients, from the Ts2Cje mouse model of DS $(\mathrm{Ts}[\mathrm{Rb}(12.17(16))] 2 \mathrm{Cje})$, and from human DS fibroblasts are enriched in APP-CTFs. The Ts2Cje model revealed that APPCTFs levels in brain EVs increase in an age-dependent manner considering a period of up to 24 months of age (133). An electron microscopy study of the neuronal endosomal system of Ts2Cje mice shows that MVBs are larger, more abundant, 
and contain a higher number of intraluminal vesicles (ILVs) compared to controls. The biochemical analysis of Ts2Cje brain endosomes revealed an enhanced content of Rab5b, Rab7, CD63, Tsg101, and Rab35, suggesting an alteration in the biogenesis and secretion of EVs $(54,134)$. The up-regulation of the endosomalEV pathway could be a homeostatic mechanism to improve endosomal dysregulation in NDs. In this regard, the brainderived EVs from 3xTgAD mouse (APP Swedish mutation, Tau P301L, and Presenilin $1 \mathrm{M} 146 \mathrm{~V}$ ) or from neuroblastoma cells show an accumulation of APP-CTFs induced by the inhibition of the $\gamma$-secretase, a molecular complex involved in $A \beta$ peptide generation. This seems to be a consequence of a defective retrograde transport of APP-CTFs from the endosomal compartments to the trans-Golgi network. The monomeric forms of APP-CTFs were mainly localized in the trans-Golgi network, whereas oligomeric forms were confined to endosomes and lysosomes, explaining the selective recovery of APP-CTFs in EVs (135).

The role of brain insulin resistance in the development of $\mathrm{AD}$ has been widely documented. Thus, the level of phosphorylation of the adaptor insulin receptor substrate (IRS)-1, a protein associated with the insulin signaling status, has been considered as a biomarker of cognitive changes. In a cohort of $\mathrm{AD}$ and diabetes mellitus, the ratio of phospho-serine 312-IRS-1 to panphospho-tyrosine-IRS-1 in neuronal EVs from plasma was found to discriminate $\mathrm{AD}$ cases from diabetic and control individuals. This insulin resistance index is higher in $\mathrm{AD}$ than in diabetic patients (136). Interestingly, the abnormal phosphorylation of IRS- 1 could predict the development of $\mathrm{AD}$ up to 10 years before clinical manifestations and correlates with cognitive performance especially in APOE4 non-carriers (137). Moreover, the pS312IRS-1/p-tyrosine-IRS-1 ratio in neuronal EVs has also been associated with the degree of brain atrophy in $\mathrm{AD}$ patients due to the volume of brain regions showing the presence of pS312IRS-1, as assessed by magnetic resonance image (138).

Vascular pathology is considered an early event of $\mathrm{AD}$ pathogenesis. The reduction of blood flow affects the content of brain-derived EVs from mice subjected to bilateral common carotid stenosis. A mass spectrometry analysis of these EVs revealed the appearance of proteins implicated in neuroprotection (glutathione peroxidase and serine protease inhibitor A3), hypoxia (agrin and myosin light chain kinase, and EGF-containing fibulin-like protein), angiogenesis (angiomotin), and AD pathogenesis (sortilin-related receptor and PACS2). Similar groups of proteins are upregulated in EVs derived from brain tissue of preclinical $\mathrm{AD}$ and $\mathrm{AD}$ with cerebrovascular disease, and in EVs derived from the serum of mice subjected to blood flow reduction to the brain (139). These findings reinforce the notion that early brain damage could be monitored in the peripheral fluids, as has been recently documented in an analysis for the identification of markers for neurocognitive impairment. The proteome of CSF -derived EVs from HIVpositive individuals with neurocognitive disorders showed an enrichment of exosomal markers like Alix, Syntenin, tetraspanins, ARF, Rab proteins, and heat-shock proteins, as well as proteins related to synapses (NPTN, NRXNs, NPTXs, and SYN1), immune/inflammatory response (ANXA, CRP, DPYSL2, ENO1, EZR, and TIMP), stress response (GST, HSPs, PARK7, PRDX, SNCA, and SNCB), mitochondrial functions (ACOT, DNM1L, DNPEP, GLUD1, RAN, and VDAC), and the blood-brain barrier (GFAP, GLUL, AGRN, AQP1, AQP4, DAG1, FBLNs, and NIDs) (140). A chronic inflammatory environment has been considered a factor underlying early events of neurodegeneration associated with the progression of dementia. Advanced glycation end products such as $\mathrm{N}$ (1-carboxymethyl)-L-lysine increase their formation during chronic inflammation, and their levels in serum-derived EVs can differentiate early to moderate $\mathrm{AD}$ (141).

The development of new and improved methodologies has enabled large-scale proteomics studies to characterize EVs obtained directly from brain tissue of animal models and $\mathrm{AD}$ patients $(142,143)$. This kind of study allows the analysis of the EV composition while preserving the tissue microenvironment and without physiological alterations associated with cell cultures. The proteomic analysis of the EVs isolated from frozen brains of mouse $A D$ models have shown that their contents change along with the development of histopathological and behavioral hallmarks of $\mathrm{AD}$. In a mouse containing mutations in the APP and PSEN1 genes (5xFAD), the EV proteome reflects complex changes in protein abundance that involve both the increase and the decrease of various components differentially at 2 and 6 months of age. For instance, at 2 months, the proteins neuromodulin (Gap43), microtubule-associated protein 2 (Map2), glia maturation factor beta $(\mathrm{Gmfb})$, and oxidation resistance protein 1 (Oxr1) are enriched in the EVs of $\mathrm{AD}$ mice. However, at 6 months, these proteins are markedly decreased in the $\mathrm{EVs}$ of $\mathrm{AD}$ mice (143). A proteomic profiling of human brain-derived EVs suggested that EV biogenesis might be altered in preclinical $\mathrm{AD}$ as indicated by an increment of MHC class I levels. In addition, EVs of preclinical cases are enriched in proteins that indicate the activation of immune response (SSBP1, PAF, and CD90), a modulation of synaptic structure (NLGN3), and a dysregulation of mitophagy (GABARAP). The progression of $\mathrm{AD}$ is characterized by an alteration in lysosome dynamics (LAMP1), an upregulation of amyloid associated proteins (APP, PrP, and ENPP2), and an induction of neurite regeneration (GAP43) (144). A similar analysis of brain-derived EVs from diagnosed $\mathrm{AD}$ cases revealed an increase of $\mathrm{A} \beta 42$ and pS396-Tau levels in $\mathrm{AD}$ samples as determined by ELISA. The label-free proteomic comparison of these EVs identified a set of differentially expressed proteins in the samples from the brain of $\mathrm{AD}$ patients, including APOE, SNCA, ANXA5, MITCH2, GPM6A, VGF, and ACTZ. Interestingly, ANXA5 shows a positive correlation with Braak stages (145). As can be appreciated from these studies, there is not an exact match of the modifications of the EV cargo. Moreover, the central core of proteins remains constant between healthy individuals and patients. This highlights the importance of the protocols employed to isolate different subpopulations of EVs and the inclusion of quantitative 
approaches to detect subtle changes in the abundance of key proteins.

\section{CURRENT ADVANCES AND CHALLENGES IN PROTEOMIC CHARACTERIZATION OF EVs}

During the last two decades there have been numerous efforts directed to determine the protein composition of several biofluids in health and disease. One of the main challenges in the molecular characterization of any biofluid is that the difference of concentrations between the most and the least abundant proteins can cover more than 10 orders of magnitude $(146,147)$. Currently, the most common and efficient method for studying complex protein mixtures is mass spectrometry; however, the limit of detection of modern instrumentation is up to five orders of the proteome dynamic range (148). This introduces a bias where only abundant proteins are confidently detected, while low-abundant proteins escape identification. Over the past few years, several strategies including labeling techniques, fractionation protocols, acquisition methods, and bioinformatic pipelines have been implemented to increase the resolution of proteomic analysis and improve quantification of proteins in clinical samples. As a result of this refinement of the mass spectrometry analysis, the compendium of plasma proteins went from 280 identified proteins in 2002 to 3,509 proteins in 2017 $(147,149)$. Nevertheless, there are still limitations in finding consistent biomarkers, especially in early phases, for $\mathrm{AD}$ and $\mathrm{PD}$. In this regard, EVs proteomics, especially from plasma, represents a promising strategy to enrich relevant molecules based on the fact that its cargo reflects the physiological state of a cell population. To further advance the discovery of biomarkers for NDs, there is a need for the standardization of protocols for EV isolation to assure reproducibility in the measurement of altered proteins.

Recent advances in the EV field have shown that there are diverse routes involved in their biogenesis, and potentially each vesicle subtype can indicate the dysfunction of a cellular process. A challenge remains in designing strategies to efficiently separate EV subtypes to identify pathogenic cargo. Most of the protocols available to isolate EVs rely on the size and density of vesicles. The proteomes of EVs from different cell sources and biofluids obtained by ultracentrifugation, precipitation, size exclusion chromatography, or ultrafiltration display components that are not necessarily EV components. The use of bottom-loaded density gradients has served to separate soluble components previously thought to be part of EV cargo such as histones, ribonucleoproteins, Argonautes, and major vault protein (51). Furthermore, the identification of proteins sensitive to trypsin digestion combined with a systems biology approach has helped to gain an insight into the real components of EVs, suggesting the presence of contaminants even after the combination of several methods of isolation (150). Particularly for CSF- and plasmaderived EVs, the elimination of non-vesicular components should be addressed in the future as a great proportion of the proteins identified in mass spectrometry studies include IgGs, coagulation factors, histones, and complement factors. The successful recovery of brain-derived EVs is one step forward in favor for the detection of low abundant proteins that could be relevant for biomarker discovery (77-79).

Parkinson's
disease


A general conclusion of the studies involving the proteomic analysis of $\mathrm{EV}$ samples from $\mathrm{AD}$ and $\mathrm{PD}$ patients is that there is a big overlap of proteins between healthy individuals and patients. Only a few dozens of proteins are exclusively found in the disease state, at least with the resolution of current methods. This highlights the importance in developing quantitative proteomic approaches with enough sensitivity that will not only register an alteration in the concentration of certain components but also allow for patient stratification $(151,152)$. A simple but powerful modification in the protocol of EV isolation accompanied by an exhaustive fractionation of the samples with nano-ultra-highperformance liquid chromatography has proved to impact the resolution of mass spectrometry analysis. Over 5,000 proteins related to the biogenesis and function of small vesicles were identified in an analysis of EV-derived from plasma obtained at low-speed centrifugation $(20,000 \times g)(153)$. These findings open the possibility to analyze other clinical samples with the same strategy and to focus on determining the tissue origin of EVs. As we detailed in this review, alterations in the phosphorylation and glycosylation of certain proteins is an aspect to be included in future mass spectrometry analysis due to its relevance in development of neurodegeneration. We envision that the study of post-translational modifications would accelerate the discovery of cellular pathways altered in the development of PD and $\mathrm{AD}$.

\section{CONCLUSIONS AND PERSPECTIVES}

There is a growing interest in the proteomic analysis of EVs due to their potential to transport biomarkers indicating the development of various chronic and degenerative diseases. The recent technical advances in proteomics have accelerated the characterization of EVs, revealing that their cargo reflect the cellular changes that occur in physiological and pathological conditions. Recently, several research groups have begun to elucidate the role of EVs in the progression of $\mathrm{AD}$ and $\mathrm{PD}$. At a cellular level, both diseases present alterations in the interaction between endosomes, lysosomes, and mitochondria, which are considered as early events of neurodegenerative processes. The dysfunction of these organelles affects the biogenesis and release of EVs by the cells in the brain. Interestingly, several genetic risk factors of NDs involve proteins implicated in the endosomal and lysosomal trafficking. The disturbance of the endolysosomal system alters normal intracellular trafficking and organelle turnover. These events are reflected in impared degradation of proteins and alterations in protein sorting (Figure 1B). Overall, the cell ability to handle cellular components and waste is compromised, altering the molecular content of EVs.

To further promote the use of EVs as biomarkers of NDs, it is necessary to develop standardized isolation protocols that ensure the quality and purity of the EV preparations. Moreover, considering that a central core of proteins remains constant between healthy and diseased individuals, the characterization of the different subpopulations of EVs is essential to identify those vesicles relevant for diagnosis purposes, and to follow the progression of NDs. During the discovery and verification phases, it will be key to correlate the EV cargo (protein, RNA, or metabolites) with relevant clinical data. The current discoveries concerning EV proteomes have already generated a list of interesting protein markers and post-translational modifications that should be corroborated in large cohorts (Figure 2). It is expected that in the following years, more candidates will be available that allow a differential diagnosis between different NDs. The EV proteins can also be useful biomarkers to follow the therapeutic response in drug trials (154). In the future, the development of new technology that provides enough sensitivity, wide dynamic range, and reliable quantification without an enrichment step of EVs would be desirable (155).

From a mechanistic point of view, a remaining challenge is to determine whether the reported changes in the content of EVs in $\mathrm{AD}$ and $\mathrm{PD}$ are a functional mechanism of communication or an indirect effect of the impairment of the management of cellular waste. This will help in the understanding of how the process of neurodegeneration starts and will give an insight into possible alternatives to halt the development of NDs. In this regard, it will be useful to perform crosssectional studies to determine the molecular content of EVs at different ages and consider peripheral sources of EVs to evaluate its influence on the function of the nervous system. An omic approach considering the association of EV cargo with blood-relevant analytes (e.g., inflammatory, metabolic) combined with brain imaging will help to build a strong diagnostic strategy.

\section{AUTHOR CONTRIBUTIONS}

RQ-B and KH-O wrote the manuscript and EM-M conceived and wrote the manuscript. All authors contributed to the article and approved the submitted version.

\section{FUNDING}

Consejo Nacional de Ciencia y Tecnología (CONACYT \#258589) and Secretaría de Educación, Ciencia, Tecnología e Innovación de la Ciudad de México (CM-SECTEI/041/2020 RECITES).

\section{ACKNOWLEDGMENTS}

This work was supported by Secretaría de Educación, Ciencia, Tecnología e Innovación de la Ciudad de México CMSECTEI/041/2020 Red colaborativa de Investigación Traslacional para el Envejecimiento Saludable de la Ciudad de México (RECITES to RQ-B), Instituto Nacional de Geriatría internal funds (DI-PI-001/2015 to RQ-B), Consejo Nacional de Ciencia y Tecnología (CONACYT, CB-258589 to EM-M), and INMEGEN internal funds (03/2018/I to EM-M). The authors are grateful to Ling Ling Lok for her careful editing. 


\section{REFERENCES}

1. Feigin VL, Vos T, Nichols E, Owolabi MO, Carroll WM, Dichgans M, et al. The global burden of neurological disorders: translating evidence into policy. Lancet Neurol. (2020) 19:255-65. doi: 10.1016/s1474-4422(19)30411-9

2. Hughes AJ, Daniel SE, Lees AJ. Improved accuracy of clinical diagnosis of Lewy body Parkinson's disease. Neurology. (2001) 57:1497-9. doi: 10.1212/wnl.57.8.1497

3. Joutsa J, Gardberg M, Roytta M, Kaasinen V. Diagnostic accuracy of parkinsonism syndromes by general neurologists. Parkinsonism Relat Disord. (2014) 20:840-4. doi: 10.1016/j.parkreldis.2014.04.019

4. Hunter CA, Kirson NY, Desai U, Cummings AK, Faries DE, Birnbaum HG. Medical costs of Alzheimer's disease misdiagnosis among US Medicare beneficiaries. Alzheimers Dement. (2015) 11:887-95. doi: 10.1016/j.jalz.2015.06.1889

5. Happich M, Kirson NY, Desai U, King S, Birnbaum HG, Reed C, et al. Excess Costs Associated with possible misdiagnosis of alzheimer's disease among patients with vascular dementia in a UK CPRD population. J Alzheimers Dis. (2016) 53:171-83. doi: 10.3233/JAD-150685

6. Van Cauwenberghe C, Van Broeckhoven C, Sleegers K. The genetic landscape of Alzheimer disease: clinical implications and perspectives. Genet Med. (2016) 18:421-30. doi: 10.1038/gim.2015.117

7. Chang D, Nalls MA, Hallgrimsdottir IB, Hunkapiller J, van der Brug M, Cai F, et al. A meta-analysis of genome-wide association studies identifies 17 new Parkinson's disease risk loci. Nat Genet. (2017) 49:1511-6. doi: 10.1038/ng.3955

8. Li YI, Wong G, Humphrey J, Raj T. Prioritizing Parkinson's disease genes using population-scale transcriptomic data. Nat Commun. (2019) 10:994. doi: 10.1038/s41467-019-08912-9

9. Poewe W, Seppi K, Tanner CM, Halliday GM, Brundin P, Volkmann J, et al. Parkinson disease. Nat Rev Dis Primers. (2017) 3:17013. doi: $10.1038 /$ nrdp. 2017.13

10. Shi M, Movius J, Dator R, Aro P, Zhao Y, Pan C, et al. Cerebrospinal fluid peptides as potential Parkinson disease biomarkers: a staged pipeline for discovery and validation. Mol Cell Proteomics. (2015) 14:544-55. doi: 10.1074/mcp.M114.040576

11. Pottiez G, Yang L, Stewart T, Song N, Aro P, Galasko DR, et al. massspectrometry-based method to quantify in parallel tau and amyloid beta 1-42 in CSF for the diagnosis of Alzheimer's disease. J Proteome Res. (2017) 16:1228-38. doi: 10.1021/acs.jproteome.6b00829

12. Hill AF. Extracellular vesicles and neurodegenerative diseases. J Neurosci. (2019) 39:9269-73. doi: 10.1523/JNEUROSCI.0147-18.2019

13. Korkut C, Ataman B, Ramachandran P, Ashley J, Barria R, Gherbesi N, et al. Trans-synaptic transmission of vesicular Wnt signals through Evi/Wntless. Cell. (2009) 139:393-404. doi: 10.1016/j.cell.2009.07.051

14. Coulter ME, Dorobantu CM, Lodewijk GA, Delalande F, Cianferani S, Ganesh VS, et al. The ESCRT-III protein CHMP1A mediates secretion of sonic hedgehog on a distinctive subtype of extracellular vesicles. Cell Rep. (2018) 24:973-86.e978. doi: 10.1016/j.celrep.2018.06.100

15. Lee SH, Shin SM, Zhong P, Kim HT, Kim DI, Kim JM, et al. Reciprocal control of excitatory synapse numbers by Wnt and Wnt inhibitor PRR7 secreted on exosomes. Nat Commun. (2018) 9:3434. doi: 10.1038/s41467-018-05858-2

16. Sharma P, Mesci P, Carromeu C, McClatchy DR, Schiapparelli L, Yates JR, et al. Exosomes regulate neurogenesis and circuit assembly. Proc Natl Acad Sci USA. (2019) 116:16086-94. doi: 10.1073/pnas.1902513116

17. Morton MC, Neckles VN, Seluzicki CM, Holmberg JC, Feliciano DM. Neonatal subventricular zone neural stem cells release extracellular vesicles that act as a microglial morphogen. Cell Rep. (2018) 23:78-89. doi: 10.1016/j.celrep.2018.03.037

18. Pais V, Pais ES. Intercellular communication by extracellular vesicles with emphasis on the roles of cordocytes in the human brain. An ultrastructural study. Ultrastruct Pathol. (2015) 39:177-86. doi: 10.3109/01913123.2014.981327

19. Balusu S, Van Wonterghem E, De Rycke R, Raemdonck K, Stremersch $\mathrm{S}$, Gevaert $\mathrm{K}$, et al. Identification of a novel mechanism of bloodbrain communication during peripheral inflammation via choroid plexus-derived extracellular vesicles. EMBO Mol Med. (2016) 8:1162-83. doi: 10.15252/emmm.201606271

20. Lachenal G, Pernet-Gallay K, Chivet M, Hemming FJ, Belly A, Bodon G, et al. Release of exosomes from differentiated neurons and its regulation by synaptic glutamatergic activity. Mol Cell Neurosci. (2011) 46:409-18. doi: 10.1016/j.mcn.2010.11.004

21. Korkut C, Li Y, Koles K, Brewer C, Ashley J, Yoshihara M, et al. Regulation of postsynaptic retrograde signaling by presynaptic exosome release. Neuron. (2013) 77:1039-46. doi: 10.1016/j.neuron.2013.01.013

22. Ashley J, Cordy B, Lucia D, Fradkin LG, Budnik V, Thomson T. Retroviruslike Gag protein Arcl binds RNA and traffics across synaptic boutons. Cell. (2018) 172:262-74.e211. doi: 10.1016/j.cell.2017.12.022

23. Pastuzyn ED, Day CE, Kearns RB, Kyrke-Smith M, Taibi AV, McCormick $\mathrm{J}$, et al. The neuronal gene Arc encodes a repurposed retrotransposon Gag protein that mediates intercellular RNA transfer. Cell. (2018) 172:27588.e218. doi: 10.1016/j.cell.2017.12.024

24. Batool A, Hill TDM, Nguyen NT, Langa E, Diviney M, Mooney C, et al. Altered biogenesis and microRNA content of hippocampal exosomes following experimental status epilepticus. Front Neurosci. (2020) 13:1404. doi: 10.3389/fnins.2019.01404

25. Yoshimura A, Adachi N, Matsuno H, Kawamata M, Yoshioka Y, Kikuchi $\mathrm{H}$, et al. The Sox2 promoter-driven CD63-GFP transgenic rat model allows tracking of neural stem cell-derived extracellular vesicles. Dis Model Mech. (2018) 11:dmm028779. doi: 10.1242/dmm.028779

26. Men Y, Yelick J, Jin S, Tian Y, Chiang MSR, Higashimori H, et al. Exosome reporter mice reveal the involvement of exosomes in mediating neuron to astroglia communication in the CNS. Nat Commun. (2019) 10:4136. doi: 10.1038/s41467-019-11534-w

27. Venturini A, Passalacqua M, Pelassa S, Pastorino F, Tedesco M, Cortese $\mathrm{K}$, et al. Exosomes from astrocyte processes: signaling to neurons. Front Pharmacol. (2019) 10:1452. doi: 10.3389/fphar.2019.01452

28. Chaudhuri AD, Dastgheyb RM, Yoo SW, Trout A, Talbot CC Jr, Hao H, et al. TNFalpha and IL-1beta modify the miRNA cargo of astrocyte shed extracellular vesicles to regulate neurotrophic signaling in neurons. Cell Death Dis. (2018) 9:363. doi: 10.1038/s41419-018-0369-4

29. Chaudhuri AD, Dasgheyb RM, DeVine LR, Bi H, Cole RN, Haughey NJ. Stimulus-dependent modifications in astrocyte-derived extracellular vesicle cargo regulate neuronal excitability. Glia. (2020) 68:128-44. doi: $10.1002 /$ glia. 23708

30. Dickens AM, Tovar-Y-Romo LB, Yoo SW, Trout AL, Bae M, Kanmogne M, et al. Astrocyte-shed extracellular vesicles regulate the peripheral leukocyte response to inflammatory brain lesions. Sci Signal. (2017) 10:eaai7696. doi: 10.1126/scisignal.aai7696

31. Bahrini I, Song JH, Diez D, Hanayama R. Neuronal exosomes facilitate synaptic pruning by up-regulating complement factors in microglia. Sci Rep. (2015) 5:7989. doi: 10.1038/srep07989

32. Beneventano M, Spampinato SF, Merlo S, Chisari M, Platania P, Ragusa M, et al. Shedding of microvesicles from microglia contributes to the effects induced by metabotropic glutamate receptor 5 activation on neuronal death. Front Pharmacol. (2017) 8:812. doi: 10.3389/fphar.2017.00812

33. Yang Y, Boza-Serrano A, Dunning CJR, Clausen BH, Lambertsen $\mathrm{KL}$, Deierborg T. Inflammation leads to distinct populations of extracellular vesicles from microglia. J Neuroinflammation. (2018) 15:168. doi: 10.1186/s12974-018-1204-7

34. Huang S, Ge X, Yu J, Han Z, Yin Z, Li Y, et al. Increased miR124-3p in microglial exosomes following traumatic brain injury inhibits neuronal inflammation and contributes to neurite outgrowth via their transfer into neurons. FASEB J. (2018) 32: 512-28. doi: 10.1096/fj.201 700673R

35. Osawa S, Kurachi M, Yamamoto H, Yoshimoto Y, Ishizaki Y. Fibronectin on extracellular vesicles from microvascular endothelial cells is involved in the vesicle uptake into oligodendrocyte precursor cells. Biochem Biophys Res Commun. (2017) 488:232-8. doi: 10.1016/j.bbrc.2017.05.049

36. Yue KY, Zhang PR, Zheng MH, Cao XL, Cao Y, Zhang YZ, et al. Neurons can upregulate Cav-1 to increase intake of endothelial cells-derived extracellular vesicles that attenuate apoptosis via miR-1290. Cell Death Dis. (2019) 10:869. doi: 10.1038/s41419-019-2100-5 
37. Kriaučiūnaite K, Kaušyle A, Pajarskiene J, Tunaitis V, Lim D, Verkhratsky $\mathrm{A}$, et al. Immortalised hippocampal astrocytes from 3xTG-AD mice fail to support $\mathrm{BBB}$ integrity in vitro: role of extracellular vesicles in glial-endothelial communication. Cell Mol Neurobiol. (2020). doi: 10.1007/s10571-020-00871-w. [Epub ahead of print].

38. Dozio V, Sanchez JC. Characterisation of extracellular vesicle-subsets derived from brain endothelial cells and analysis of their protein cargo modulation after TNF exposure. J Extracell Vesicles. (2017) 6:1302705. doi: 10.1080/20013078.2017.1302705

39. Couch Y, Akbar N, Roodselaar J, Evans MC, Gardiner C, Sargent I, et al. Circulating endothelial cell-derived extracellular vesicles mediate the acute phase response and sickness behaviour associated with CNS inflammation. Sci Rep. (2017) 7:9574. doi: 10.1038/s41598-017-09710-3

40. Morad G, Carman CV, Hagedorn EJ, Perlin JR, Zon LI, Mustafaoglu $\mathrm{N}$, et al. Tumor-derived extracellular vesicles breach the intact blood-brain barrier via transcytosis. ACS Nano. (2019) 13:13853-65. doi: 10.1021/acsnano.9b04397

41. García-Romero N, Carrión-Navarro J, Esteban-Rubio S, Lázaro-Ibáñez E, Peris-Celda M, Alonso MM, et al. DNA sequences within gliomaderived extracellular vesicles can cross the intact blood-brain barrier and be detected in peripheral blood of patients. Oncotarget. (2017) 8:1416-28. doi: 10.18632/oncotarget.13635

42. Wei Z, Batagov AO, Schinelli S, Wang J, Wang Y, El Fatimy R, et al. Coding and noncoding landscape of extracellular RNA released by human glioma stem cells. Nat Commun. (2017) 8:1145. doi: 10.1038/s41467-017-01196-x

43. Figueroa JM, Skog J, Akers J, Li H, Komotar R, Jensen R, et al. Detection of wild-Type EGFR amplification and EGFRvIII mutation in CSF-derived extracellular vesicles of glioblastoma patients. Neuro-oncology. (2017) 19:1494-502. doi: 10.1093/neuonc/nox085

44. Mallawaaratchy DM, Hallal S, Russell B, Ly L, Ebrahimkhani S, Wei H, et al. Comprehensive proteome profiling of glioblastoma-derived extracellular vesicles identifies markers for more aggressive disease. J Neurooncol. (2017) 131:233-44. doi: 10.1007/s11060-016-2298-3

45. Goetzl EJ, Boxer A, Schwartz JB, Abner EL, Petersen RC, Miller $\mathrm{BL}$, et al. Altered lysosomal proteins in neural-derived plasma exosomes in preclinical Alzheimer disease. Neurology. (2015) 85:40-7. doi: 10.1212/WNL.0000000000001702

46. Goetzl EJ, Elahi FM, Mustapic M, Kapogiannis D, Pryhoda M, Gilmore A, et al. Altered levels of plasma neuron-derived exosomes and their cargo proteins characterize acute and chronic mild traumatic brain injury. FASEB J. (2019) 33:5082-8. doi: 10.1096/fj.201 802319R

47. Muraoka S, Jedrychowski MP, Tatebe H, DeLeo AM, Ikezu S, Tokuda $\mathrm{T}$, et al. Proteomic profiling of extracellular vesicles isolated from cerebrospinal fluid of former national football league players at risk for chronic traumatic encephalopathy. Front Neurosci. (2019) 13:1059. doi: 10.3389/fnins.2019.01059

48. Deng J, Koutras C, Donnelier J, Alshehri M, Fotouhi M, Girard $\mathrm{M}$, et al. Neurons export extracellular vesicles enriched in cysteine string protein and misfolded protein cargo. Sci Rep. (2017) 7:956. doi: 10.1038/s41598-017-01115-6

49. Miranda AM, Lasiecka ZM, Xu Y, Neufeld J, Shahriar S, Simoes S, et al. Neuronal lysosomal dysfunction releases exosomes harboring APP Cterminal fragments and unique lipid signatures. Nat Commun. (2018) 9:291. doi: 10.1038/s41467-017-02533-w

50. Kowal J, Arras G, Colombo M, Jouve M, Morath JP, Primdal-Bengtson $\mathrm{B}$, et al. Proteomic comparison defines novel markers to characterize heterogeneous populations of extracellular vesicle subtypes. Proc Natl Acad Sci USA. (2016) 113:E968-977. doi: 10.1073/pnas.1521230113

51. Jeppesen DK, Fenix AM, Franklin JL, Higginbotham JN, Zhang Q, Zimmerman LJ, et al. Reassessment of exosome composition. Cell. (2019) 177:428-45.e418. doi: 10.1016/j.cell.2019.02.029

52. Nixon RA, Yang DS. Autophagy failure in Alzheimer's diseaselocating the primary defect. Neurobiol Dis. (2011) 43:38-45. doi: 10.1016/j.nbd.2011.01.021

53. Escudero CA, Lazo OM, Galleguillos C, Parraguez JI, Lopez-Verrilli MA, Cabeza C, et al. The p75 neurotrophin receptor evades the endolysosomal route in neuronal cells, favouring multivesicular bodies specialised for exosomal release. J Cell Sci. (2014) 127(Pt 9):1966-79. doi: $10.1242 /$ jcs. 141754

54. D’Acunzo P, Hargash T, Pawlik M, Goulbourne CN, Pérez-González R, Levy E. Enhanced generation of intraluminal vesicles in neuronal late endosomes in the brain of a Down syndrome mouse model with endosomal dysfunction. Dev Neurobiol. (2019) 79:656-63. doi: 10.1002/dneu. 22708

55. Peng KY, Pérez-González R, Alldred MJ, Goulbourne CN, Morales-Corraliza J, Saito M, et al. Apolipoprotein E4 genotype compromises brain exosome production. Brain. (2019) 142:163-75. doi: 10.1093/brain/awy289

56. Ngolab J, Trinh I, Rockenstein E, Mante M, Florio J, Trejo M, et al. Brain-derived exosomes from dementia with Lewy bodies propagate a-synuclein pathology. Acta Neuropathol Commun. (2017) 5:46. doi: 10.1186/s40478-017-0445-5

57. Sardar Sinha M, Ansell-Schultz A, Civitelli L, Hildesjö C, Larsson M, Lannfelt L, et al. Alzheimer's disease pathology propagation by exosomes containing toxic amyloid-beta oligomers. Acta Neuropathol. (2018) 136:4156. doi: 10.1007/s00401-018-1868-1

58. Aulston B, Liu Q, Mante M, Florio J, Rissman RA, Yuan SH. Extracellular vesicles isolated from familial Alzheimer's disease neuronal cultures induce aberrant tau phosphorylation in the wild-type mouse brain. J Alzheimers Dis. (2019) 72:575-85. doi: 10.3233/JAD-190656

59. Laulagnier K, Javalet C, Hemming FJ, Chivet M, Lachenal G, Blot B, et al. Amyloid precursor protein products concentrate in a subset of exosomes specifically endocytosed by neurons. Cell Mol Life Sci. (2018) 75:757-73. doi: 10.1007/s00018-017-2664-0

60. Murphy KE, Gysbers AM, Abbott SK, Spiro AS, Furuta A, Cooper A, et al. Lysosomal-associated membrane protein 2 isoforms are differentially affected in early Parkinson's disease. Mov Disord. (2015) 30:1639-47. doi: $10.1002 / \mathrm{mds} .26141$

61. Song P, Trajkovic K, Tsunemi T, Krainc D. Parkin modulates endosomal organization and function of the endo-lysosomal pathway. J Neurosci. (2016) 36:2425-37. doi: 10.1523/JNEUROSCI.2569-15.2016

62. Tsunemi T, Hamada K, Krainc D. ATP13A2/PARK9 regulates secretion of exosomes and alpha-synuclein. J Neurosci. (2014) 34:15281-7. doi: 10.1523/JNEUROSCI.1629-14.2014

63. Sackmann V, Sinha MS, Sackmann C, Civitelli L, Bergström J, AnsellSchultz A, et al. Inhibition of nSMase2 reduces the transfer of oligomeric $\alpha$-synuclein irrespective of hypoxia. Front Mol Neurosci. (2019) 12:200. doi: 10.3389/fnmol.2019.00200

64. Fraser KB, Moehle MS, Daher JP, Webber PJ, Williams JY, Stewart CA, et al. LRRK2 secretion in exosomes is regulated by 14-3-3. Hum Mol Genet. (2013) 22:4988-5000. doi: 10.1093/hmg/ddt346

65. Wang S, Liu Z, Ye T, Mabrouk OS, Maltbie T, Aasly J, et al. Elevated LRRK2 autophosphorylation in brain-derived and peripheral exosomes in LRRK2 mutation carriers. Acta Neuropathol Commun. (2017) 5:86. doi: 10.1186/s40478-017-0492-y

66. Fraser KB, Moehle MS, Alcalay RN, West AB, Consortium LC. Urinary LRRK2 phosphorylation predicts parkinsonian phenotypes in G2019S LRRK2 carriers. Neurology. (2016) 86:994-9. doi: 10.1212/WNL.0000000000002436

67. Mahul-Mellier AL, Burtscher J, Maharjan N, Weerens L, Croisier M, Kuttler F, et al. The process of Lewy body formation, rather than simply alphasynuclein fibrillization, is one of the major drivers of neurodegeneration. Proc Natl Acad Sci USA. (2020) 117:4971-82. doi: 10.1073/pnas.1913904117

68. Braak H, Del Tredici K, Rüb U, De Vos RAI, Jansen Steur ENH, Braak E. Staging of brain pathology related to sporadic Parkinson's disease. Neurobiol Aging. (2003) 24:197-211. doi: 10.1016/S0197-4580(02)00065-9

69. Shannon KM, Keshavarzian A, Mutlu E, Dodiya HB, Daian D, Jaglin JA, et al. Alpha-synuclein in colonic submucosa in early untreated Parkinson's disease. Mov Disord. (2012) 27:709-15. doi: 10.1002/mds.23838

70. Tsutsumi R, Hori Y, Seki T, Kurauchi Y, Sato M, Oshima M, et al. Involvement of exosomes in dopaminergic neurodegeneration by microglial activation in midbrain slice cultures. Biochem Biophys Res Commun. (2019) 511:427-33. doi: 10.1016/j.bbrc.2019.02.076

71. Wu F, Xu HD, Guan JJ, Hou YS, Gu JH, Zhen XC, et al. Rotenone impairs autophagic flux and lysosomal functions in Parkinson's disease. Neuroscience. (2015) 284:900-11. doi: 10.1016/j.neuroscience.2014.11.004 
72. Demers-Lamarche J, Guillebaud G, Tlili M, Todkar K, Belanger N, Grondin $\mathrm{M}$, et al. Loss of mitochondrial function impairs lysosomes. J Biol Chem. (2016) 291:10263-76. doi: 10.1074/jbc.M115.695825

73. Matheoud D, Sugiura A, Bellemare-Pelletier A, Laplante A, Rondeau C, Chemali $\mathrm{M}$, et al. Parkinson's disease-related proteins PINK1 and Parkin repress mitochondrial antigen presentation. Cell. (2016) 166:314-27. doi: 10.1016/j.cell.2016.05.039

74. Minakaki G, Menges S, Kittel A, Emmanouilidou E, Schaeffner I, Barkovits K, et al. Autophagy inhibition promotes SNCA/alphasynuclein release and transfer via extracellular vesicles with a hybrid autophagosome-exosome-like phenotype. Autophagy. (2018) 14:98-119. doi: 10.1080/15548627.2017.1395992

75. El-Agnaf OMA, Salem SA, Paleologou KE, Curran MD, Gibson MJ, Court JA, et al. Detection of oligomeric forms of $\alpha$-synuclein protein in human plasma as a potential biomarker for Parkinson's disease. FASEB J. (2006) 20:419-25. doi: 10.1096/fj.03-1449com

76. Stuendl A, Kunadt M, Kruse N, Bartels C, Moebius W, Danzer KM, et al. Induction of $\alpha$-synuclein aggregate formation by CSF exosomes from patients with Parkinson's disease and dementia with Lewy bodies. Brain. (2016) 139:481-94. doi: 10.1093/brain/awv346

77. Shi M, Liu C, Cook TJ, Bullock KM, Zhao Y, Ginghina C, et al. Plasma exosomal alpha-synuclein is likely CNS-derived and increased in Parkinson's disease. Acta Neuropathol. (2014) 128:639-50. doi: 10.1007/s00401-014-1314-y

78. Shi M, Kovac A, Korff A, Cook TJ, Ginghina C, Bullock KM, et al. CNS tau efflux via exosomes is likely increased in Parkinson's disease but not in Alzheimer's disease. Alzheimers Dement. (2016) 12:1125-31. doi: 10.1016/j.jalz.2016.04.003

79. Ohmichi T, Mitsuhashi M, Tatebe H, Kasai T, Ali El-Agnaf OM, Tokuda T. Quantification of brain-derived extracellular vesicles in plasma as a biomarker to diagnose Parkinson's and related diseases. Parkinsonism Relat Disord. (2019) 61:82-7. doi: 10.1016/j.parkreldis.2018.11.021

80. Jiang C, Hopfner F, Katsikoudi A, Hein R, Catli C, Evetts S, et al. Serum neuronal exosomes predict and differentiate Parkinson's disease from atypical parkinsonism. J Neurol Neurosurg Psychiatr. (2020) 91:720-9. doi: 10.1136/jnnp-2019-322588

81. Ho DH, Yi S, Seo H, Son I, Seol W. Increased DJ-1 in urine exosome of Korean males with Parkinson's disease. Biomed Res Int. (2014) 2014:704678. doi: 10.1155/2014/704678

82. Cao Z, Wu Y, Liu G, Jiang Y, Wang X, Wang Z, et al. alpha-Synuclein in salivary extracellular vesicles as a potential biomarker of Parkinson's disease. Neurosci Lett. (2019) 696:114-20. doi: 10.1016/j.neulet.2018.12.030

83. Rani K, Mukherjee R, Singh E, Kumar S, Sharma V, Vishwakarma $P$, et al. Neuronal exosomes in saliva of Parkinson's disease patients: a pilot study. Parkinsonism Relat Disord. (2019) 67:21-3. doi: 10.1016/j.parkreldis.2019.09.008

84. Boerger M, Funke S, Leha A, Roser AE, Wuestemann AK, Maass F, et al. Proteomic analysis of tear fluid reveals disease-specific patterns in patients with Parkinson's disease-a pilot study. Parkinsonism Relat Disord. (2019) 63:3-9. doi: 10.1016/j.parkreldis.2019.03.001

85. Papadopoulos VE, Nikolopoulou G, Antoniadou I, Karachaliou A, Arianoglou G, Emmanouilidou E, et al. Modulation of $\beta$-glucocerebrosidase increases $\alpha$-synuclein secretion and exosome release in mouse models of Parkinson's disease | Human Molecular Genetics | Oxford Academic. Hum Mol Genet. (2018) 27:1696-710. doi: 10.1093/hmg/ddy075

86. Thomas RE, Vincow ES, Merrihew GE, MacCoss MJ, Davis MY, Pallanck LJ. Glucocerebrosidase deficiency promotes protein aggregation through dysregulation of extracellular vesicles. PLoS Genet. (2018) 14:e1007694. doi: 10.1371/journal.pgen.1007694

87. Osellame LD, Rahim AA, Hargreaves IP, Gegg ME, Richard-Londt A, Brandner S, et al. Mitochondria and quality control defects in a mouse model of Gaucher disease-links to Parkinson's disease. Cell Metab. (2013) 17:941-53. doi: 10.1016/j.cmet.2013.04.014

88. Rocha EM, De Miranda B, Sanders LH. Alpha-synuclein: Pathology, mitochondrial dysfunction and neuroinflammation in Parkinson's disease. Neurobiol Dis. (2018) 109:249-57. doi: 10.1016/j.nbd.2017.04.004s

89. Ho DH, Je AR, Lee H, Son I, Kweon HS, Kim HG, et al. LRRK2 kinase activity induces mitochondrial fission in microglia via Drp1 and modulates neuroinflammation. Exp Neurobiol. (2018) 27:171-80. doi: 10.5607/en.2018.27.3.171

90. Fraser KB, Rawlins AB, Clark RG, Alcalay RN, Standaert DG, Liu N, et al. Ser(P)-1292 LRRK2 in urinary exosomes is elevated in idiopathic Parkinson's disease. Mov Disord. (2016) 31:1543-50. doi: 10.1002/mds.26686

91. Zhao Z-H, Chen Z-T, Zhou R-L, Zhang X, Ye Q-Y, Wang Y-Z. Increased DJ-1 and $\alpha$-synuclein in plasma neural-derived exosomes as potential markers for Parkinson's disease. Front Aging Neurosci. (2019) 10:438. doi: 10.3389/fnagi.2018.00438

92. Tomlinson PR, Zheng Y, Fischer R, Heidasch R, Gardiner C, Evetts S, et al. Identification of distinct circulating exosomes in Parkinson's disease. Ann Clin Transl Neurol. (2015) 2:353-61. doi: 10.1002/acn3.175

93. Jiang R, Rong C, Ke R, Meng S, Yan X, Ke H, et al. Differential proteomic analysis of serum exosomes reveals alterations in progression of Parkinson disease. Medicine (Baltimore). (2019) 98:e17478. doi: 10.1097/MD.0000000000017478

94. Kitamura Y, Kojima M, Kurosawa T, Sasaki R, Ichihara S, Hiraku $\mathrm{Y}$, et al. Proteomic profiling of exosomal proteins for blood-based biomarkers in Parkinson's disease. Neuroscience. (2018) 392:121-8. doi: 10.1016/j.neuroscience.2018.09.017

95. Lamontagne-Proulx J, St-Amour I, Labib R, Pilon J, Denis HL, Cloutier N, et al. Portrait of blood-derived extracellular vesicles in patients with Parkinson's disease. Neurobiol Dis. (2019) 124:163-75. doi: 10.1016/j.nbd.2018.11.002

96. Wang S, Kojima K, Mobley JA, West AB. Proteomic analysis of urinary extracellular vesicles reveal biomarkers for neurologic disease. EBioMedicine. (2019) 45:351-61. doi: 10.1016/j.ebiom.2019.06.021

97. Picca A, Guerra F, Calvani R, Marini F, Biancolillo A, Landi G, et al. Mitochondrial signatures in circulating extracellular vesicles of older adults with Parkinson's disease: results from the EXosomes in PArkiNson's Disease (EXPAND) study. J Clin Med. (2020) 9:504. doi: 10.3390/jcm9020504

98. Yuyama K, Sun H, Sakai S, Mitsutake S, Okada M, Tahara H, et al. Decreased amyloid- $\beta$ pathologies by intracerebral loading of glycosphingolipidenriched exosomes in Alzheimer model mice. J Biol Chem. (2014) 289:2448898. doi: 10.1074/jbc.M114.577213

99. Yuyama K, Sun H, Usuki S, Sakai S, Hanamatsu H, Mioka T, et al. A potential function for neuronal exosomes: sequestering intracerebral amyloid- $\beta$ peptide. FEBS Lett. (2015) 589:84-8. doi: 10.1016/j.febslet.2014.11.027

100. Cui GH, Wu J, Mou FF, Xie WH, Wang FB, Wang QL, et al. Exosomes derived from hypoxia-preconditioned mesenchymal stromal cells ameliorate cognitive decline by rescuing synaptic dysfunction and regulating inflammatory responses in APP/PS1 mice. FASEB J. (2018) 32:654-68. doi: 10.1096/fj.201700600R

101. de Godoy MA, Saraiva LM, de Carvalho LRP, Vasconcelos-dos-Santos A, Beiral HJV, Ramos AB, et al. Mesenchymal stem cells and cell-derived extracellular vesicles protect hippocampal neurons from oxidative stress and synapse damage induced by amyloid-oligomers. J Biol Chem. (2018) 293:1957-75. doi: 10.1074/jbc.M117.807180

102. Wang SS, Jia J, Wang Z. Mesenchymal stem cell-derived extracellular vesicles suppresses iNOS expression and ameliorates neural impairment in Alzheimer's disease mice. J Alzheimers Dis. (2018) 61:1005-13. doi: 10.3233/JAD-170848

103. Fauré J, Lachenal G, Court M, Hirrlinger J, Chatellard-Causse C, Blot B, et al. Exosomes are released by cultured cortical neurones. Mol Cell Neurosci. (2006) 31:642-8. doi: 10.1016/j.mcn.2005.12.003

104. Falker C, Hartmann A, Guett I, Dohler F, Altmeppen H, Betzel C, et al. Exosomal cellular prion protein drives fibrillization of amyloid beta and counteracts amyloid beta-mediated neurotoxicity. J Neurochem. (2016) 137:88-100. doi: 10.1111/jnc.13514

105. An K, Klyubin I, Kim Y, Jung JH, Mably AJ, O’Dowd ST, et al. Exosomes neutralize synaptic-plasticity-disrupting activity of $\mathrm{A} \beta$ assemblies in vivo. Mol Brain. (2013) 6:47. doi: 10.1186/1756-6606-6-47

106. Zheng T, Pu J, Chen Y, Mao Y, Guo Z, Pan H, et al. Plasma exosomes spread and cluster around $\beta$-amyloid plaques in an animal model of Alzheimer's disease. Front Aging Neurosci. (2017) 9:12. doi: 10.3389/fnagi.2017.00012

107. Joshi P, Turola E, Ruiz A, Bergami A, Libera DD, Benussi L, et al. Microglia convert aggregated amyloid- $\beta$ into neurotoxic forms through the shedding of microvesicles. Cell Death Differ. (2014) 21:582-93. doi: 10.1038/cdd.2013.180 
108. Gouwens LK, Ismail MS, Rogers VA, Zeller NT, Garrad EC, Amtashar FS, et al. A $\beta 42$ protofibrils interact with and are trafficked through microglial-derived microvesicles. ACS Chem Neurosci. (2018) 9:1416-25. doi: 10.1021/acschemneuro.8b00029

109. Gireud-Goss M, Reyes S, Tewari R, Patrizz A, Howe MD, Kofler J, et al. The ubiquitin ligase UBE4B regulates amyloid precursor protein ubiquitination, endosomal trafficking, and amyloid beta42 generation and secretion. $\mathrm{Mol}$ Cell Neurosci. (2020) 108:103542. doi: 10.1016/j.mcn.2020.103542

110. Pacheco-Quinto J, Clausen D, Pérez-González R, Peng H, Meszaros $\mathrm{A}$, Eckman $\mathrm{CB}$, et al. Intracellular metalloprotease activity controls intraneuronal $A \beta$ aggregation and limits secretion of $A \beta$ via exosomes. FASEB Journal. (2019) 33:3758-71. doi: 10.1096/fj.201801319R

111. Fiandaca MS, Kapogiannis D, Mapstone M, Boxer A, Eitan E, Schwartz JB, et al. Identification of preclinical Alzheimer's disease by a profile of pathogenic proteins in neurally derived blood exosomes: a case-control study. Alzheimers Dement. (2015) 11:600-7.e601. doi: 10.1016/j.jalz.2014.06.008

112. Perrotte M, Haddad M, Le Page A, Frost EH, Fulöp T, Ramassamy C. Profile of pathogenic proteins in total circulating extracellular vesicles in mild cognitive impairment and during the progression of Alzheimer's disease. Neurobiol Aging. (2020) 86:102-11. doi: 10.1016/j.neurobiolaging.2019.10.010

113. Goetzl EJ, Boxer A, Schwartz JB, Abner EL, Petersen RC, Miller BL, et al. Low neural exosomal levels of cellular survival factors in Alzheimer's disease. Ann Clin Transl Neurol. (2015) 2:769-73. doi: 10.1002/acn3.211

114. Saman S, Kim W, Raya M, Visnick Y, Miro S, Saman S, et al. Exosome-associated tau is secreted in tauopathy models and is selectively phosphorylated in cerebrospinal fluid in early Alzheimer disease. J Biol Chem. (2012) 287:3842-9. doi: 10.1074/jbc.M111.277061

115. Asai H, Ikezu S, Tsunoda S, Medalla M, Luebke J, Haydar T, et al. Depletion of microglia and inhibition of exosome synthesis halt tau propagation. Nat Neurosci. (2015) 18:1584-93. doi: 10.1038/nn.4132

116. Wang JKT, Langfelder P, Horvath S, Palazzolo MJ. Exosomes and homeostatic synaptic plasticity are linked to each other and to Huntington's, Parkinson's, and other neurodegenerative diseases by database-enabled analyses of comprehensively curated datasets. Front Neurosci. (2017) 11:149. doi: 10.3389/fnins.2017.00149

117. Polanco JC, Scicluna BJ, Hill AF, Götz J. Extracellular vesicles isolated from the brains of $\mathrm{rTg} 4510$ mice seed tau protein aggregation in a threshold-dependent manner. J Biol Chem. (2016) 291:12445-66. doi: 10.1074/jbc.M115.709485

118. Lambert JC, Ibrahim-Verbaas CA, Harold D, Naj AC, Sims R, Bellenguez C, et al. Meta-analysis of 74,046 individuals identifies 11 new susceptibility loci for Alzheimer's disease. Nat Genet. (2013) 45:1452-8. doi: 10.1038/ng.2802

119. Crotti A, Sait HR, McAvoy KM, Estrada K, Ergun A, Szak S, et al. BIN1 favors the spreading of Tau via extracellular vesicles. Sci Rep. (2019) 9:1-20. doi: 10.1038/s41598-019-45676-0

120. Guix FX, Corbett GT, Cha DJ, Mustapic M, Liu W, Mengel D, et al. Detection of aggregation-competent tau in neuron-derived extracellular vesicles. Int J Mol Sci. (2018) 19:663. doi: 10.3390/ijms19030663

121. Podvin S, Jones A, Liu Q, Aulston B, Ransom L, Ames J, et al. Dysregulation of exosome cargo by mutant tau expressed in human-induced pluripotent stem cell (iPSC) neurons revealed by proteomics analyses. Mol Cell Proteomics. (2020) 19:1017-34. doi: 10.1074/mcp.RA120.002079

122. Lin YT, Seo J, Gao F, Feldman HM, Wen HL, Penney J, et al. APOE4 causes widespread molecular and cellular alterations associated with Alzheimer's disease phenotypes in human iPSC-derived brain cell types. Neuron. (2018) 98:1141-54.e1147. doi: 10.1016/j.neuron.2018.05.008

123. Nikitidou E, Khoonsari PE, Shevchenko G, Ingelsson M, Kultima K, Erlandsson A. Increased release of apolipoprotein E in extracellular vesicles following amyloid- $\beta$ protofibril exposure of neuroglial co-cultures. J Alzheimers Dis. (2017) 60:305-21. doi: 10.3233/JAD-170278

124. Sollvander S, Nikitidou E, Brolin R, Soderberg L, Sehlin D, Lannfelt L, et al. Accumulation of amyloid-beta by astrocytes result in enlarged endosomes and microvesicle-induced apoptosis of neurons. Mol Neurodegener. (2016) 11:38. doi: 10.1186/s13024-016-0098-z

125. Musunuri S, Khoonsari PE, Mikus M, Wetterhall M, Häggmark-Mänberg A, Lannfelt L, et al. Increased levels of extracellular microvesicle markers and decreased levels of endocytic/exocytic proteins in the Alzheimer's disease brain. J Alzheimers Dis. (2016) 54:1671-86. doi: 10.3233/JAD-160271

126. Eitan E, Hutchison ER, Marosi K, Comotto J, Mustapic M, Nigam SM, et al. Extracellular vesicle-associated a $\beta$ mediates trans-neuronal bioenergetic and $\mathrm{Ca}^{2+}$-handling deficits in alzheimer's disease models. NPJ Aging Mech Dis. (2016) 2:16019. doi: 10.1038/npjamd.2016.19

127. Frenkel-Pinter M, Shmueli MD, Raz C, Yanku M, Zilberzwige S, Gazit E, et al. Interplay between protein glycosylation pathways in Alzheimer's disease. Sci Adv. (2017) 3:e1601576. doi: 10.1126/sciadv. 1601576

128. Louwersheimer E, Cohn-Hokke PE, Pijnenburg YA, Weiss MM, Sistermans EA, Rozemuller AJ, et al. Rare genetic variant in SORL1 may increase penetrance of Alzheimer's disease in a family with several generations of APOE-varepsilon4 homozygosity. J Alzheimers Dis. (2017) 56:63-74. doi: 10.3233/JAD-160091

129. Royo F, Cossio U, Ruiz de Angulo A, Llop J, Falcon-Perez JM. Modification of the glycosylation of extracellular vesicles alters their biodistribution in mice. Nanoscale. (2019) 11:1531-7. doi: 10.1039/c8nr03900c

130. Nishida-Aoki N, Tominaga N, Kosaka N, Ochiya T. Altered biodistribution of deglycosylated extracellular vesicles through enhanced cellular uptake. $J$ Extracell Vesicles. (2020) 9:1713527. doi: 10.1080/20013078.2020.1713527

131. Winston CN, Aulston B, Rockenstein EM, Adame A, Prikhodko O, Dave KN, et al. Neuronal exosome-derived human tau is toxic to recipient mouse neurons in vivo. J Alzheimers Dis. (2019) 67:541-53. doi: 10.3233/JAD-180776

132. Baker S, Polanco JC, Götz J. Extracellular Vesicles Containing P301L Mutant Tau Accelerate pathological tau phosphorylation and oligomer formation but do not seed mature neurofibrillary tangles in ALZ17 mice. J Alzheimers Dis. (2016) 54:1207-17. doi: 10.3233/JAD-160371

133. Pérez-González R, Gauthier SA, Sharma A, Miller C, Pawlik M, Kaur G, et al. A pleiotropic role for exosomes loaded with the amyloid $\beta$ precursor protein carboxyl-terminal fragments in the brain of Down syndrome patients. Neurobiol Aging. (2019) 84:26-32. doi: 10.1016/j.neurobiolaging.2019.07.016

134. Gauthier SA, Pérez-González R, Sharma A, Huang FK, Alldred MJ, Pawlik M, et al. Enhanced exosome secretion in Down syndrome brain-a protective mechanism to alleviate neuronal endosomal abnormalities. Acta Neuropathol Commun. (2017) 5:65. doi: 10.1186/s40478-017-0466-0

135. Lauritzen I, Bécot A, Bourgeois A, Pardossi-Piquard R, Biferi MG, Barkats M, et al. Targeting $\gamma$-secretase triggers the selective enrichment of oligomeric APP-CTFs in brain extracellular vesicles from Alzheimer cell and mouse models. Transl Neurodegener. (2019) 8:35. doi: 10.1186/s40035-0190176-6

136. Kapogiannis D, Boxer A, Schwartz JB, Abner EL, Biragyn A, Masharani U, et al. Dysfunctionally phosphorylated type 1 insulin receptor substrate in neural-derived blood exosomes of preclinical Alzheimer's disease. FASEB J. (2015) 29:589-96. doi: 10.1096/fj.14-262048

137. Mustapic M, Tran J, Craft S, Kapogiannis D. Extracellular vesicle biomarkers track cognitive changes following intranasal insulin in Alzheimer's disease. $J$ Alzheimers Dis. (2019) 69:489-98. doi: 10.3233/JAD-180578

138. Mullins RJ, Mustapic M, Goetzl EJ, Kapogiannis D. Exosomal biomarkers of brain insulin resistance associated with regional atrophy in Alzheimer's disease. Hum Brain Mapp. (2017) 38:1933-40. doi: 10.1002/hbm.23494

139. Gallart-Palau X, Serra A, Hase Y, Tan CF, Chen CP, Kalaria RN, et al. Brain-derived and circulating vesicle profiles indicate neurovascular unit dysfunction in early Alzheimer's disease. Brain Pathol. (2019) 29:593-605. doi: 10.1111/bpa.12699

140. Guha D, Lorenz DR, Misra V, Chettimada S, Morgello S, Gabuzda D. Proteomic analysis of cerebrospinal fluid extracellular vesicles reveals synaptic injury, inflammation, and stress response markers in HIV patients with cognitive impairment. J Neuroinflammation. (2019) 16:254.doi: 10.1186/s12974-019-1617-y

141. Haddad M, Perrotte M, Landri S, Lepage A, Fülöp T, Ramassamy C. Circulating and extracellular vesicles levels of N-(1-carboxymethyl)-L-lysine (CML) differentiate early to moderate Alzheimer's disease. J Alzheimers Dis. (2019) 69:751-62. doi: 10.3233/JAD-181272

142. Vella LJ, Scicluna BJ, Cheng L, Bawden EG, Masters CL, Ang CS, et al. A rigorous method to enrich for exosomes from brain tissue. J Extracell Vesicles. (2017) 6:1348885. doi: 10.1080/20013078.2017.1348885 
143. Hurwitz SN, Sun L, Cole KY, Ford CR III, Olcese JM, Meckes DG Jr. An optimized method for enrichment of whole brain-derived extracellular vesicles reveals insight into neurodegenerative processes in a mouse model of Alzheimer's disease. J Neurosci Methods. (2018) 307:210-20. doi: 10.1016/j.jneumeth.2018.05.022

144. Gallart-Palau X, Guo X, Serra A, Sze SK. Alzheimer's disease progression characterized by alterations in the molecular profiles and biogenesis of brain extracellular vesicles. Alzheimer's Res Ther. (2020) 12:54. doi: 10.1186/s13195-020-00623-4

145. Muraoka S, DeLeo AM, Sethi MK, Yukawa-Takamatsu K, Yang Z, Ko $\mathrm{J}$, et al. Proteomic and biological profiling of extracellular vesicles from Alzheimer's disease human brain tissues. Alzheimers Dement. (2020) 16:896907. doi: 10.1002/alz.12089

146. Zubarev RA. The challenge of the proteome dynamic range and its implications for in-depth proteomics. Proteomics. (2013) 13:723-6. doi: $10.1002 /$ pmic. 201200451

147. Schwenk JM, Omenn GS, Sun Z, Campbell DS, Baker MS, Overall CM, et al. The human plasma proteome draft of 2017: building on the human plasma PeptideAtlas from mass spectrometry and complementary assays. J Proteome Res. (2017) 16:4299-310. doi: 10.1021/acs.jproteome. $7 \mathrm{~b} 00467$

148. Beri J, Rosenblatt MM, Strauss E, Urh M, Bereman MS. Reagent for evaluating liquid chromatography-tandem mass spectrometry (LC-MS/MS) performance in bottom-up proteomic experiments. Anal Chem. (2015) 87:11635-40. doi: 10.1021/acs.analchem. 5 b04121

149. Anderson NL, Anderson NG. The human plasma proteome: history, character, and diagnostic prospects. Mol Cell Proteomics. (2002) 1:845-67. doi: 10.1074/mcp.r200007-mcp200

150. Choi D, Go G, Kim DK, Lee J, Park SM, Di Vizio D, et al. Quantitative proteomic analysis of trypsin-treated extracellular vesicles to identify the real-vesicular proteins. J Extracell Vesicles. (2020) 9:1757209. doi: 10.1080/20013078.2020.1757209
151. McGurk KA, Dagliati A, Chiasserini D, Lee D, Plant D, Baricevic-Jones I, et al. The use of missing values in proteomic data-independent acquisition mass spectrometry to enable disease activity discrimination. Bioinformatics. (2020) 36:2217-23. doi: 10.1093/bioinformatics/btz898

152. Zheng $\mathrm{X}, \mathrm{Xu} \mathrm{K}$, Zhou B, Chen $\mathrm{T}$, Huang $\mathrm{Y}, \mathrm{Li}$ Q, et al. A circulating extracellular vesicles-based novel screening tool for colorectal cancer revealed by shotgun and data-independent acquisition mass spectrometry. J Extracell Vesicles. (2020) 9:1750202. doi: 10.1080/20013078.2020.1750202

153. Whitham M, Parker BL, Friedrichsen M, Hingst JR, Hjorth M, Hughes WE, et al. Extracellular vesicles provide a means for tissue crosstalk during exercise. Cell Metabolism. (2018) 27:237-251.e234. doi: 10.1016/j.cmet.2017.12.001

154. Athauda D, Gulyani S, Karnati HK, Li Y, Tweedie D, Mustapic M, et al. Utility of neuronal-derived exosomes to examine molecular mechanisms that affect motor function in patients with Parkinson disease: a secondary analysis of the exenatide-PD trial. JAMA Neurol. (2019) 76:420-9. doi: 10.1001/jamaneurol.2018.4304

155. Mabrouk OS, Chen S, Edwards AL, Yang M, Hirst WD, Graham DL. Quantitative measurements of LRRK2 in human cerebrospinal fluid demonstrates increased levels in G2019S patients. Front Neurosci. (2020) 14:526. doi: 10.3389/fnins.2020.00526

Conflict of Interest: The authors declare that the research was conducted in the absence of any commercial or financial relationships that could be construed as a potential conflict of interest.

Copyright (๑) 2020 Quiroz-Baez, Hernández-Ortega and Martínez-Martínez. This is an open-access article distributed under the terms of the Creative Commons Attribution License (CC BY). The use, distribution or reproduction in other forums is permitted, provided the original author(s) and the copyright owner(s) are credited and that the original publication in this journal is cited, in accordance with accepted academic practice. No use, distribution or reproduction is permitted which does not comply with these terms. 